\title{
Vibration Control by Exploiting Nonlinear Influence in the Frequency Domain
}

Xingiian Jing

Additional information is available at the end of the chapter

http://dx.doi.org/10.5772/45795

\section{Introduction}

In the control theory of linear systems, system transfer function provides a coordinate-free and equivalent description for system dynamic characteristics, by which it is convenient to conduct analysis and design. Therefore, frequency domain methods are commonly used by engineers and widely applied in engineering practice. However, although the analysis and design of linear systems in the frequency domain have been well established, the frequency domain analysis for nonlinear systems is not straightforward. Nonlinear systems usually have very complicated output frequency characteristics and dynamic behaviour such as harmonics, inter-modulation, chaos and bifurcation. Investigation and understanding of these nonlinear phenomena in the frequency domain are far from full development. Frequency domain methods for nonlinear analysis have been investigated for many years. There are several different approaches to the analysis and design for nonlinear systems, such as describing functions [5, 13], harmonic balance [18], and frequency domain methods developed from the absolute stability theory [10], for example the well-known Popov circle theorem $[12,21]$ etc. Investigation of nonlinear systems in the frequency domain can also be done based on the Volterra series expansion theory $[11,15,16,19,20]$. There are a large class of nonlinear systems which have a convergent Volterra series expansion [2, 17]. For this class of nonlinear systems, referred to as Volterra systems, the generalized frequency response function (GFRF) was defined in [4], which is similar to the transfer function of linear systems. To obtain the GFRFs for Volterra systems described by nonlinear differential equations, the probing method can be used [16]. Once the GRFRs are obtained for a practical system, system output spectrum can then be evaluated [9]. These form a fundamental basis for the analysis of nonlinear Volterra systems in the frequency domain and provide an elegant and useful method for the frequency domain analysis of a class of nonlinear systems. Many techniques developed (e.g. the GFRFs) can be regarded as an important extension of frequency domain theories for linear systems to nonlinear cases. 
In this study, understanding of nonlinearity in the frequency domain is investigated from a novel viewpoint for Volterra systems. The system output spectrum is shown to be an alternating series with respect to some model parameters under certain conditions. This property has great significance in that the system output spectrum can therefore be easily suppressed by tuning the corresponding parameters. This provides a novel insight into the nonlinear influence in a system. The sufficient (and necessary) conditions in which the output spectrum can be transformed into an alternating series are studied. These results are illustrated by two example studies which investigated a single degree of freedom (SDOF) springdamping system with a cubic nonlinear damping. The results established in this study demonstrate a novel characteristic of the nonlinear influence in the frequency domain, and provide a novel insight into the analysis and design of nonlinear vibration control systems.

The chapter is organised as follows. Section 2 provides a detailed background of this study. The novel nonlinear characteristic and its influence are discussed in Section 3. Section 4 gives a sufficient and necessary condition under which system output spectrum can be transformed into an alternating series. A conclusion is given in Section 5. A nomenclature section which explains the main notations used in this paper is given in Appendix A.

\section{Frequency response functions of nonlinear systems}

There are a class of nonlinear systems for which the input-output relationship can be sufficiently approximated by a Volterra series (of a maximum order $N$ ) around the zero equilibrium as $[2,17]$

$$
y(t)=\sum_{n=1}^{N} \int_{-\infty}^{\infty} \cdots \int_{-\infty}^{\infty} h_{n}\left(\tau_{1}, \cdots, \tau_{n}\right) \prod_{i=1}^{n} u\left(t-\tau_{i}\right) d \tau_{i}
$$

where $h_{n}\left(\tau_{1}, \cdots, \tau_{n}\right)$ is the $n$ th-order Volterra kernel which is a real valued function of $\tau_{1}, \cdots, \tau_{n}$. For the same class of nonlinear systems, it can also be modelled by the following nonlinear differential equation (NDE)

$$
\sum_{m=1}^{M} \sum_{p=0}^{m} \sum_{k_{1}, k_{m}=0}^{K} c_{p, m-p}\left(k_{1}, \cdots, k_{m}\right) \prod_{i=1}^{p} \frac{d^{k_{i}} y(t)}{d t^{k_{i}}} \prod_{i=p+1}^{m} \frac{d^{k_{i}} u(t)}{d t^{k_{i}}}=0
$$

where $\left.\frac{d^{k} x(t)}{d t^{k}}\right|_{k=0}=x(t), \sum_{k_{1}, k_{p+q}=0}^{K}(\cdot)=\sum_{k_{1}=0}^{K}(\cdot) \cdots \sum_{k_{p+q}=0}^{K}(\cdot), M$ is the maximum degree of nonlinearity in terms of $y(t)$ and $u(t)$, and $K$ is the maximum order of the derivative. In this model, the parameters such as $c 0,1($.$) and c 1,0($.$) are referred to as linear parameters corresponding to$ coefficients of linear terms in the model, i.e., $\frac{d^{k} y(t)}{d t^{k}}$ and $\frac{d^{k} u(t)}{d t^{k}}$ for $k=0,1, \ldots, K$; and $c_{p, q}(\cdot)$ for $p+q>1$ are referred to as nonlinear parameters corresponding to nonlinear terms in the model of the form $\prod_{i=1}^{p} \frac{d^{k_{i}} y(t)}{d t^{k_{i}}} \prod_{i=p+1}^{p+q} \frac{d^{k_{i}} u(t)}{d t^{k_{i}}}$, e.g., $y(t)^{p} u(t)^{q}$. The value $p+q$ is referred to as the nonlinear degree of parameter $c_{p, q}(\cdot)$. 
By using the probing method [16], a recursive algorithm for the computation of the $n$ thorder generalized frequency response function (GFRF) for the NDE model (2) is provided in [1]. Therefore, the output spectrum of model (2) can be evaluated as [9]

$$
Y(j \omega)=\sum_{n=1}^{N} \frac{1}{\sqrt{n}(2 \pi)^{n-1}} \int_{\omega_{1}+\cdots+\omega_{n}=\omega} H_{n}\left(j \omega_{1}, \cdots, j \omega_{n}\right) \prod_{i=1}^{n} U\left(j \omega_{i}\right) d \sigma_{\omega}
$$

which is truncated at the largest order $N$ and where,

$$
H_{n}\left(j \omega_{1}, \cdots, j \omega_{n}\right)=\int_{-\infty}^{\infty} \cdots \int_{-\infty}^{\infty} h_{n}\left(\tau_{1}, \cdots, \tau_{n}\right) \exp \left(-j\left(\omega_{1} \tau_{1}+\cdots+\omega_{n} \tau_{n}\right)\right) d \tau_{1} \cdots d \tau_{n}
$$

is known as the $n$ th-order GFRF defined in [4], and $h_{n}\left(\tau_{1}, \cdots, \tau_{n}\right)$ is the $n$ th-order Volterra kernel introduced in (1). When the system input is a multi-tone function described by

$$
u(t)=\sum_{i=1}^{\bar{K}}\left|F_{i}\right| \cos \left(\omega_{i} t+\angle F_{i}\right)
$$

(where $F_{i}$ is a complex number, $\angle F_{i}$ is the argument, $\left|F_{i}\right|$ is the modulus, and $\bar{K}$ is a positive integer), the system output frequency response can be evaluated as [9]:

$$
Y(j \omega)=\sum_{n=1}^{N} \frac{1}{2^{n}} \sum_{\omega_{k_{1}}+\cdots+\omega_{k_{n}}=\omega} H_{n}\left(j \omega_{k_{1}}, \cdots, j \omega_{k_{n}}\right) F\left(\omega_{k_{1}}\right) \cdots F\left(\omega_{k_{n}}\right)
$$

where $F\left(\omega_{k_{i}}\right)$ can be explicitly written as $F\left(\omega_{k_{i}}\right)=\left|F_{\left|k_{i}\right|}\right| e^{j\left\langle F_{k_{i}}\right| \cdot \operatorname{sig}\left(k_{i}\right)}$ for $k_{i} \in\{ \pm 1, \cdots, \pm \bar{K}\}$ in stead of the form in [9], $\operatorname{sgn}(a)=\left\{\begin{array}{ll}1 & a \geq 0 \\ -1 & a<0\end{array}\right.$, and $\omega_{k_{i}} \in\left\{ \pm \omega_{1}, \cdots, \pm \omega_{\bar{K}}\right\}$.

In order to explicitly reveal the relationship between model parameters and the frequency response functions above, the parametric characteristics of the GFRFs and output spectrum are studied in [6]. The $n$ th-order GFRF can then be expressed into a more straightforward polynomial form as

$$
H_{n}\left(j \omega_{1}, \cdots, j \omega_{n}\right)=C E\left(H_{n}\left(j \omega_{1}, \cdots, j \omega_{n}\right)\right) \cdot f_{n}\left(j \omega_{1}, \cdots, j \omega_{n}\right)
$$

where $C E\left(H_{n}\left(j \omega_{1}, \cdots, j \omega_{n}\right)\right)$ is referred to as the parametric characteristic of the $n$ th-order GFRF $H_{n}\left(j \omega_{1}, \cdots, j \omega_{n}\right)$, which can be recursively determined as

$$
C E\left(H_{n}\left(j \omega_{1}, \cdots, j \omega_{n}\right)\right)=C_{0, n} \oplus\left(\underset{q=1}{\oplus} \oplus_{p=1}^{n-1} C_{p, q} \otimes C E\left(H_{n-q-p+1}(\cdot)\right)\right) \oplus\left(\underset{p=2}{\oplus} C_{p, 0} \otimes C E\left(H_{n-p+1}(\cdot)\right)\right)
$$

with terminating condition $C E\left(H_{1}\left(j \omega_{i}\right)\right)=1$. Note that $C E$ is a new operator with two operations " $\otimes$ " and " $\oplus$ " defined in $[6,7]$ (the definition of $C E$ can be referred to Appendix 
B and more detailed discussions in [22]), and $C_{p, q}$ is a vector consisting of all the $(p+q)$ th degree nonlinear parameters, i.e.,

$$
C_{p, q}=\left[c_{p, q}(0, \cdots, 0), c_{p, q}(0, \cdots, 1), \cdots, c_{p, q}(\underbrace{K, \cdots, K}_{p+q=m})\right]
$$

In Equation (8), $f_{n}\left(j \omega_{1}, \cdots, j \omega_{n}\right)$ is a complex valued vector with the same dimension as $C E\left(H_{n}\left(j \omega_{1}, \cdots, j \omega_{n}\right)\right)$. In [7], a mapping function $\varphi_{n}\left(C E\left(H_{n}(\cdot)\right) ; \omega_{1}, \cdots, \omega_{n}\right)$ from the parametric characteristic $C E\left(H_{n}\left(j \omega_{1}, \cdots, j \omega_{n}\right)\right)$ to its corresponding correlative function $f_{n}\left(j \omega_{1}, \cdots, j \omega_{n}\right)$ is established as

$$
\begin{aligned}
& \varphi_{n(\bar{s})}\left(c_{p_{0}, q_{0}}(\cdot) c_{p_{1}, q_{1}}(\cdot) \cdots c_{p_{k}, q_{k}}(\cdot) ; \omega_{l(1)} \cdots \omega_{l(n(\bar{s}))}\right)
\end{aligned}
$$

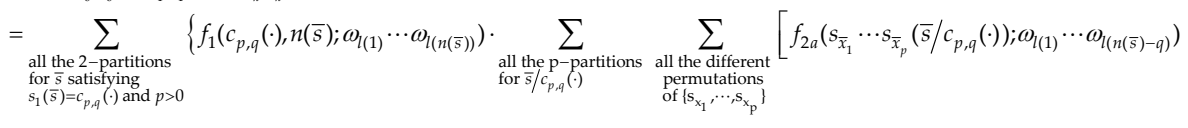

$$
\begin{aligned}
& \left.\left.\cdot \prod_{i=1}^{p} \varphi_{n\left(s_{\bar{x}_{i}}\left(\bar{s} / c_{p, q}(\cdot)\right)\right)}\left(s_{\bar{x}_{i}}\left(\bar{s} / c_{p, q}(\cdot)\right) ; \omega_{l(\bar{X}(i)+1)} \cdots \omega_{l\left(\bar{X}(i)+n\left(s_{x_{i}}\left(\bar{s} / c_{p, q}(\cdot)\right)\right)\right)}\right)\right]\right\}
\end{aligned}
$$

where the terminating condition is $k=0$ and $\varphi_{1}\left(1 ; \omega_{i}\right)=H_{1}\left(j \omega_{i}\right)$ (which is the transfer function when all nonlinear parameters are zero), $\left\{s_{\bar{x}_{1}}, \cdots s_{\bar{x}_{p}}\right\}$ is a permutation of $\left\{s_{x_{1}}, \cdots s_{x_{p}}\right\}$, $\omega_{l(1)} \cdots \omega_{l(n(\bar{s}))}$ represents the frequency variables involved in the corresponding functions, $l(i)$ for $i=1 \ldots n(\bar{s})$ is a positive integer representing the index of the frequency variables, $\bar{s}=c_{p_{0}, q_{0}}(\cdot) c_{p_{1}, q_{1}}(\cdot) \cdots c_{p_{k}, q_{k}}(\cdot), n\left(s_{x}(\bar{s})\right)=\sum_{i=1}^{x}\left(p_{i}+q_{i}\right)-x+1, x$ is the number of the parameters in $s_{x}, \sum_{i=1}^{x}\left(p_{i}+q_{i}\right)$ is the sum of the subscripts of all the parameters in $s_{x}$. Moreover,

$$
\begin{gathered}
\bar{X}(i)=\sum_{j=1}^{i-1} n\left(s_{\bar{x}_{j}}\left(\bar{s} / c_{p q}(\cdot)\right)\right) \\
L_{n}(j \varpi)=-\sum_{k_{1}=0}^{K} c_{1,0}\left(k_{1}\right)(j \varpi)^{k_{1}} \quad \forall \varpi \in R \\
f_{1}\left(c_{p, q}(\cdot), n(\bar{s}) ; \omega_{l(1)} \cdots \omega_{l(n(\bar{s}))}\right)=\left(\prod_{i=1}^{q}\left(j \omega_{l(n(\bar{s})-q+i)}\right)^{k_{p+i}} / L_{n(\bar{s})}\left(j \sum_{i=1}^{n(\bar{s})} \omega_{l(i)}\right)\right. \\
f_{2 a}\left(s_{\bar{x}_{1}} \cdots s_{\bar{x}_{p}}\left(\bar{s} / c_{p, q}(\cdot)\right) ; \omega_{l(1)} \cdots \omega_{l(n(\bar{s})-q)}\right)=\prod_{i=1}^{p}\left(j \omega_{l(\bar{X}(i)+1)}+\cdots+j \omega_{l\left(\bar{X}(i)+n\left(s_{\bar{x}_{i}}\left(\bar{s} / c_{p q}(\cdot)\right)\right)\right)}\right)^{k_{i}}
\end{gathered}
$$

The mapping function $\varphi_{n}\left(C E\left(H_{n}(\cdot)\right) ; \omega_{1}, \cdots, \omega_{n}\right)$ enables the complex valued function $f_{n}\left(j \omega_{1}, \cdots, j \omega_{n}\right)$ to be analytically and directly determined in terms of the first order GFRF and nonlinear parameters. Therefore, the $n$ th-order GFRF can directly be written into a more 
straightforward and meaningful polynomial function in terms of the first order GFRF and model parameters by using the mapping function $\varphi_{n}\left(C E\left(H_{n}(\cdot)\right) ; \omega_{1}, \cdots, \omega_{n}\right)$ as

$$
H_{n}\left(j \omega_{1}, \cdots, j \omega_{n}\right)=C E\left(H_{n}\left(j \omega_{1}, \cdots, j \omega_{n}\right)\right) \cdot \varphi_{n}\left(C E\left(H_{n}(\cdot)\right) ; \omega_{1}, \cdots, \omega_{n}\right)
$$

Using (10), Equation (3) can be written as

$$
Y(j \omega)=\sum_{n=1}^{N} C E\left(H_{n}\left(j \omega_{1}, \cdots, j \omega_{n}\right)\right) \cdot \bar{F}_{n}(j \omega)
$$

where $\bar{F}_{n}(j \omega)=\frac{1}{\sqrt{n}(2 \pi)^{n-1}} \int_{\omega_{1}+\cdots+\omega_{n}=\omega} \varphi_{n}\left(C E\left(H_{n}(\cdot) ; \omega_{1}, \cdots, \omega_{n}\right) \cdot \prod_{i=1}^{n} U\left(j \omega_{i}\right) d \sigma_{\omega}\right.$. Similarly, Equation (6) can be written as

$$
Y(j \omega)=\sum_{n=1}^{N} C E\left(H_{n}\left(j \omega_{k_{1}}, \cdots, j \omega_{k_{n}}\right)\right) \cdot \tilde{F}_{n}(\omega)
$$

where $\tilde{F}_{n}(j \omega)=\frac{1}{2^{n}} \sum_{\omega_{k_{1}}+\cdots+\omega_{k_{n}}=\omega} \varphi_{n}\left(C E\left(H_{n}(\cdot)\right) ; \omega_{k_{1}}, \cdots, \omega_{k_{n}}\right) \cdot F\left(\omega_{k_{1}}\right) \cdots F\left(\omega_{k_{n}}\right)$. Note that the expressions for output spectrum above are all truncated at the largest order $N$. The significance of the expressions in (10-11) is that, the explicit relationship between any model parameters and the frequency response functions can be demonstrated clearly and thus it is convenient to be used for system analysis and design.

Example 1. Consider a simple example to demonstrate the results above. Suppose all the other nonlinear parameters in (2) are zero except $c_{1,1}(1,1), c_{0,2}(1,1), c_{2,0}(1,1)$. For convenience, $C_{1,1}(1,1)$ is written as $c_{1,1}$ and so on. Consider the parametric characteristic of $H_{3}($.$) , which can$ easily be derived from (8),

$$
\begin{aligned}
& C E\left(H_{3}\left(j \omega_{1}, \cdots, j \omega_{3}\right)\right) \\
& \quad=C_{0,3} \oplus C_{1,1} \otimes C_{0,2} \oplus C_{1,1}^{2} \oplus C_{1,1} \otimes C_{2,0} \oplus C_{2,1} \oplus C_{1,2} \oplus C_{2,0} \otimes C_{0,2} \oplus C_{2,0}^{2} \oplus C_{3,0} \\
& \quad=C_{1,1} \otimes C_{0,2} \oplus C_{1,1}^{2} \oplus C_{1,1} \otimes C_{2,0} \oplus C_{2,0} \otimes C_{0,2} \oplus C_{2,0}^{2}
\end{aligned}
$$

Note that $\mathrm{C}_{1,1}=\mathcal{C}_{1,1}, \mathrm{C}_{0,2}=\mathcal{C}_{0,2}, \mathrm{C}_{2,0}=\mathrm{C} 2,0$. Thus,

$$
C E\left(H_{3}\left(j \omega_{1}, \cdots, j \omega_{3}\right)\right)=\left[c_{1,1} c_{0,2}, c_{1,1}^{2}, c_{1,1} c_{2,0}, c_{2,0} c_{0,2}, c_{2,0} c_{1,1}, c_{2,0}^{2}\right]
$$

Using (9abc), the correlative functions of each term in $C E\left(H_{3}\left(j \omega_{1}, \cdots, j \omega_{3}\right)\right)$ can all be obtained. For example, for the term $c_{1,1} c_{0,2}$, it can be derived directly from ( $9 \mathrm{abc}$ ) that

$$
\begin{aligned}
& \varphi_{n(\bar{s})}\left(c_{1,1}(\cdot) c_{0,2}(\cdot) ; \omega_{l(1)} \cdots \omega_{l(n(\bar{s}))}\right)=\varphi_{3}\left(c_{1,1}(\cdot) c_{0,2}(\cdot) ; \omega_{1} \cdots \omega_{3}\right) \\
& =f_{1}\left(c_{1,1}(\cdot), 3 ; \omega_{1} \cdots \omega_{3}\right) \cdot f_{2 a}\left(s_{1}\left(c_{1,1}(\cdot) c_{0,2}(\cdot) / c_{1,1}(\cdot)\right) ; \omega_{1}, \omega_{2}\right) \cdot \varphi_{2}\left(s_{1}\left(c_{0,2}(\cdot)\right) ; \omega_{1}, \omega_{2}\right) \\
& =f_{1}\left(c_{1,1}(\cdot), 3 ; \omega_{1} \cdots \omega_{3}\right) \cdot f_{2 a}\left(c_{0,2}(\cdot) ; \omega_{1}, \omega_{2}\right) \cdot \varphi_{2}\left(c_{0,2}(\cdot) ; \omega_{1}, \omega_{2}\right) \\
& =\frac{j \omega_{3}}{L_{3}\left(j \omega_{1}+\cdots+j \omega_{3}\right)} \cdot\left(j \omega_{1}+j \omega_{2}\right) \cdot \frac{j \omega_{1} j \omega_{2}}{L_{2}\left(j \omega_{1}+j \omega_{2}\right)}=\frac{j \omega_{1} j \omega_{2} j \omega_{3}\left(j \omega_{1}+j \omega_{2}\right)}{L_{3}\left(j \omega_{1}+\cdots+j \omega_{3}\right) L_{2}\left(j \omega_{1}+j \omega_{2}\right)}
\end{aligned}
$$


Proceed with the process above, the whole correlative function of $C E\left(H_{3}\left(j \omega_{1}, \cdots, j \omega_{3}\right)\right)$ can be obtained, and then $(10-11 \mathrm{ab})$ can be determined. This demonstrates a new way to analytically compute the high order GFRFs, and the final results can directly be written into a polynomial form as (10-11ab), for example in this case

$$
\begin{aligned}
& H_{3}\left(j \omega_{1}, \cdots, j \omega_{3}\right)=\left[c_{1,1} c_{0,2}, c_{1,1}^{2}, c_{1,1} c_{2,0}, c_{2,0} c_{0,2}, c_{2,0} c_{1,1}, c_{2,0}^{2}\right] \cdot \varphi_{3}\left(C E\left(H_{3}\left(j \omega_{1}, \cdots, j \omega_{3}\right)\right) ; \omega_{1}, \cdots, \omega_{3}\right) \\
& =c_{1,1} c_{0,2} \cdot \varphi_{3}\left(c_{1,1} c_{0,2} ; \omega_{1}, \cdots, \omega_{3}\right)+c_{1,1}^{2} \cdot \varphi_{3}\left(c_{1,1}^{2} ; \omega_{1}, \cdots, \omega_{3}\right)+\ldots+c_{2,0}^{2} \cdot \varphi_{3}\left(c_{2,0}^{2} ; \omega_{1}, \cdots, \omega_{3}\right)
\end{aligned}
$$

As discussed in [7], it can be seen from Equations (10-11ab) and Example 1 that the mapping function $\varphi_{n}\left(C E\left(H_{n}(\cdot)\right) ; \omega_{1}, \cdots, \omega_{n}\right)$ can facilitate the frequency domain analysis of nonlinear systems such that the relationship between the frequency response functions and model parameters, and the relationship between the frequency response functions and $H_{1}\left(j \omega_{l(1)}\right)$ can be demonstrated explicitly, and some new properties of the GFRFs and output spectrum can be revealed. In practice, the output spectrum of a nonlinear system can be expanded as a power series with respect to a specific model parameter of interest by using (11ab) for $\mathrm{N} \rightarrow \infty$. The nonlinear effect on system output spectrum incurred by this model parameter which may represents the physical characteristic of a structural unit in the system can then be analysed and designed by studying this power series in the frequency domain. Note that the fundamental properties of this power series (e.g. convergence) are to a large extent dominated by the properties of its coefficients, which are explicitly determined by the mapping function $\varphi_{n}\left(C E\left(H_{n}(\cdot)\right) ; \omega_{1}, \cdots, \omega_{n}\right)$. Thus studying the properties of this power series is now equivalent to studying the properties of the mapping function $\varphi_{n}\left(C E\left(H_{n}(\cdot)\right) ; \omega_{1}, \cdots, \omega_{n}\right)$. Therefore, the mapping function $\varphi_{n}\left(C E\left(H_{n}(\cdot)\right) ; \omega_{1}, \cdots, \omega_{n}\right)$ introduced above provides an important and significant technique for this frequency domain analysis to study the nonlinear influence on system output spectrum.

In this study, a novel property of the nonlinear influence on system output spectrum is revealed by using the new mapping function $\varphi_{n}\left(C E\left(H_{n}(\cdot)\right) ; \omega_{1}, \cdots, \omega_{n}\right)$ and frequency response functions defined in Equations (10-11). It is shown that the nonlinear terms in a system can drive the system output spectrum to be an alternating series under certain conditions when the system subjects to a sinusoidal input, and the system output spectrum is shown to have some interesting properties in engineering practice when it can be expanded into an alternating series with respect to a specific model parameter of interest. This provides a novel insight into the nonlinear effect incurred by nonlinear terms in a nonlinear system to the system output spectrum.

\section{Alternating phenomenon in the output spectrum and its influence}

The alternating phenomena and its influence are discussed in this section to point out the significance of this novel property, and then the conditions under which system output spectrum can be expressed into an alternating series are studied in the following section. 
For any nonlinear parameter (simply denoted by $c$ ) in model (2), the output spectrum (11ab) can be expanded with respect to this parameter into a power series as

$$
Y(j \omega)=F_{0}(j \omega)+c F_{1}(j \omega)+c^{2} F_{2}(j \omega)+\cdots+c^{\rho} F_{\rho}(j \omega)+\cdots
$$

Note that when $c$ represents a nonlinearity from input terms, Equation (12) may be a finite series; in other cases, it is definitely an infinite series, and if only the first $\rho$ terms in the series (12) are considered, there is a truncation error denoted by $o(\rho)$. As demonstrated in Example $1, F_{i}(j \omega)$ for $\mathrm{i}=0,1,2, \ldots$ are some scalar frequency functions and can be obtained from $\bar{F}_{i}(j \omega)$ or $\widetilde{F}_{i}(j \omega)$ in $(11 \mathrm{a}, \mathrm{b})$ by using the mapping function $\varphi_{n}\left(C E\left(H_{n}(\cdot)\right) ; \omega_{1}, \cdots, \omega_{n}\right)$. Clearly, $F_{i}(j \omega)$ dominates the fundamental properties of this power series such as convergence. Thus these properties of this power series can be revealed by studying the property of $\varphi_{n}\left(C E\left(H_{n}(\cdot)\right) ; \omega_{1}, \cdots, \omega_{n}\right)$. This will be discussed more in the next section. In this section, the alternating phenomenon of this power series and its influence are discussed.

For any $v \in \mathbb{C}$, define an operator as

$$
\operatorname{sgn}_{c}(v)=\left[\operatorname{sgn}_{r}(\operatorname{Re}(v)) \quad \operatorname{sgn}_{r}(\operatorname{Im}(v))\right]
$$

where $\operatorname{sgn}_{r}(x)=\left\{\begin{array}{ll}+1 & x>0 \\ 0 & x=0 \\ -1 & x<0\end{array}\right.$ for $x \in \mathbb{R}$.

Definition 1 (Alternating series). Consider a power series of form (12) with $c>0$. If $\operatorname{sgn}_{c}\left(F_{i}(j \omega)\right)=-\operatorname{sgn}_{c}\left(F_{i+1}(j \omega)\right)$ for $\mathrm{i}=0,1,2,3, \ldots$, then the series is an alternating series.

The series (12) can be written into two series as

$$
\begin{aligned}
& Y(j \omega)=\operatorname{Re}(Y(j \omega))+j(\operatorname{Im}(Y(j \omega))) \\
& =\operatorname{Re}\left(F_{0}(j \omega)\right)+c \operatorname{Re}\left(F_{1}(j \omega)\right)+c^{2} \operatorname{Re}\left(F_{2}(j \omega)\right)+\cdots+c^{\rho} \operatorname{Re}\left(F_{\rho}(j \omega)\right)+\cdots \\
& \quad+j\left(\operatorname{Im}\left(F_{0}(j \omega)\right)+c \operatorname{Im}\left(F_{1}(j \omega)\right)+c^{2} \operatorname{Im}\left(F_{2}(j \omega)\right)+\cdots+c^{\rho} \operatorname{Im}\left(F_{\rho}(j \omega)\right)+\cdots\right)
\end{aligned}
$$

From definition 1, if $Y(j \omega)$ is an alternating series, then $\operatorname{Re}(Y(j \omega))$ and $\operatorname{Im}(Y(j \omega))$ are both alternating. When (12) is an alternating series, there are some interesting properties summarized in Theorem 1. Denote

$$
Y(j \omega)_{1 \rightarrow \rho}=F_{0}(j \omega)+c F_{1}(j \omega)+c^{2} F_{2}(j \omega)+\cdots+c^{\rho} F_{\rho}(j \omega)
$$

Theorem 1. Suppose (12) is an alternating series at a $\omega\left(\in \mathbb{R}_{+}\right)$for $c>0$, then:

(1) if there exist $T>0$ and $R>0$ such that for $i>T$

$$
\min \left\{-\frac{\operatorname{Re}\left(F_{i}(j \omega)\right)}{\operatorname{Re}\left(F_{i+1}(j \omega)\right)},-\frac{\operatorname{Im}\left(F_{i}(j \omega)\right)}{\operatorname{Im}\left(F_{i+1}(j \omega)\right)}\right\}>R
$$


then (12) has a radius of convergence $R$, the truncation error for a finite order $\rho>T$ is $|o(\rho)| \leq c^{\rho+1}\left|F_{\rho+1}(j \omega)\right|$, and for all $n \geq 0$,

$$
|Y(j \omega)| \in \Pi_{n}=\left[\left|Y(j \omega)_{1 \rightarrow T+2 n+1}\right|,\left|Y(j \omega)_{1 \rightarrow T+2 n}\right|\right] \text { and } \Pi_{n+1} \subset \Pi_{n} ;
$$

(2) $|Y(j \omega)|^{2}=Y(j \omega) Y(-j \omega)$ is also an alternating series with respect to parameter c; Furthermore, $|Y(j \omega)|^{2}=Y(j \omega) Y(-j \omega)$ is alternating only if $\operatorname{Re}(Y(j \omega))$ is alternating;

(3) there exists a constant $\bar{c}>0$ such that $\frac{\partial|Y(j \omega)|}{\partial c}<0$ for $0<c<\bar{c}$.

\section{Proof. See Appendix C. $\square$}

The first point in Theorem 1 shows that only if there exists a positive constant $R>0$, the series must be convergent under $0<c<R$, its truncation error and limit value can therefore be easily evaluated. The other two points of Theorem 1 imply that the magnitude of an alternating series can be suppressed by choosing a proper value for the parameter $c$. Therefore, once the system output spectrum can be expressed into an alternating series with respect to a model parameter (say $c$ ), it is easier to find a proper value for $c$ such that the output spectrum is convergent, and the magnitude can be suppressed. Moreover, it is also shown that the lowest limit of the magnitude of the output spectrum that can be reached is larger than $\left|Y(j \omega)_{1 \rightarrow T+1}\right|$ and the truncation error of the output spectrum is less than the absolute value of the term of the largest order at the truncated point.

Example 2. Consider a single degree of freedom (SDOF) spring-damping system with a cubic nonlinear damping which can be described by the following differential equation

$$
m \ddot{y}=-k_{0} y-B \dot{y}-c \dot{y}^{3}+u(t)
$$

Note that $k_{0}$ represents the spring characteristic, $B$ the damping characteristic and $c$ is the cubic nonlinear damping characteristic. This system is a simple case of NDE model (2) and can be written into the form of NDE model with $M=3, K=2$, $c_{1,0}(2)=m, c_{1,0}(1)=B, c_{1,0}(0)=k_{0}, c_{3,0}(111)=c, c_{0,1}(0)=-1$ and all the other parameters are zero.

Note that there is only one nonlinear term in the output in this case, the $n$ th-order GFRF for system (15) can be derived according to the algorithm in [1], which can be recursively determined as

$$
\begin{gathered}
H_{n}\left(j \omega_{1}, \cdots, j \omega_{n}\right)=\frac{c_{3,0}(1,1,1) H_{n, 3}\left(j \omega_{1}, \cdots, j \omega_{n}\right)}{L_{n}\left(j \omega_{1}+\cdots+j \omega_{n}\right)} \\
H_{n, 3}(\cdot)=\sum_{i=1}^{n-2} H_{i}\left(j \omega_{1}, \cdots, j \omega_{i}\right) H_{n-i, 2}\left(j \omega_{i+1}, \cdots, j \omega_{n}\right)\left(j \omega_{1}+\cdots+j \omega_{i}\right)
\end{gathered}
$$




$$
H_{n, 1}\left(j \omega_{1}, \cdots, j \omega_{n}\right)=H_{n}\left(j \omega_{1}, \cdots, j \omega_{n}\right)\left(j \omega_{1}+\cdots+j \omega_{n}\right)
$$

Proceeding with the recursive computation above, it can be seen that $H_{n}\left(j \omega_{1}, \cdots, j \omega_{n}\right)$ is a polynomial of $c_{3,0}(111)$, and substituting these equations above into (11) gives another polynomial for the output spectrum. By using the relationship (10) and the mapping function $\varphi_{n}\left(C E\left(H_{n}(\cdot)\right) ; \omega_{1}, \cdots, \omega_{n}\right)$, these results can be obtained directly as follows.

For simplicity, let $u(t)=F_{d} \sin (\Omega t) \quad\left(F_{d}>0\right)$. Then $F\left(\omega_{k_{l}}\right)=-j k_{l} F_{d}$, for $k_{l}= \pm 1, \omega_{k_{l}}=k_{l} \Omega$, and $l=1, \cdots, n$ in (11b). By using (8) or Proposition 5 in [6], it can be obtained that

$$
C E\left(H_{2 n+1}\left(j \omega_{1}, \cdots, j \omega_{2 n+1}\right)\right)=\left(c_{3,0}(1,1,1)\right)^{n} \text { and } C E\left(H_{2 n}\left(j \omega_{1}, \cdots, j \omega_{2 n}\right)\right)=0 \text { for } \mathrm{n}=0,1,2,3, \ldots
$$

Therefore, for $n=0,1,2,3, \ldots$

$$
H_{2 n+1}\left(j \omega_{1}, \cdots, j \omega_{2 n+1}\right)=c^{n} \cdot \varphi_{2 n+1}\left(C E\left(H_{2 n+1}(\cdot)\right) ; \omega_{1}, \cdots, \omega_{2 n+1}\right) \text { and } H_{2 n}\left(j \omega_{1}, \cdots, j \omega_{2 n}\right)=0
$$

Then the output spectrum at frequency $\Omega$ can be computed as ( $N$ is the largest order after truncated)

$$
Y(j \Omega)=\sum_{n=0}^{\lfloor N-1 / 2\rfloor} c^{n} \cdot \widetilde{F}_{2 n+1}(\Omega)+\cdots
$$

where $\widetilde{F}_{2 n+1}(j \Omega)$ can be computed as

$$
\begin{aligned}
\tilde{F}_{2 n+1}(j \Omega) & =\frac{1}{2^{2 n+1}} \sum_{\omega_{k_{1}}+\cdots+\omega_{k_{2 n+1}}=\Omega} \varphi_{2 n+1}\left(\operatorname{CE}\left(H_{2 n+1}(\cdot)\right) ; \omega_{k_{1}}, \cdots, \omega_{k_{2 n+1}}\right) \cdot\left(-j F_{d}\right)^{2 n+1} \cdot k_{1} k_{2} \cdots k_{2 n+1} \\
& =\frac{1}{2^{2 n+1}} \sum_{\omega_{k_{1}}+\cdots+\omega_{k_{2 n+1}}=\Omega} \varphi_{2 n+1}\left(C E\left(H_{2 n+1}(\cdot)\right) ; \omega_{k_{1}}, \cdots, \omega_{k_{2 n+1}}\right) \cdot(-1)^{n+1} j\left(F_{d}\right)^{2 n+1} \cdot(-1)^{n} \\
& =-j\left(\frac{F_{d}}{2}\right)^{2 n+1} \sum_{\omega_{k_{1}}+\cdots+\omega_{k_{2 n+1}}=\Omega} \varphi_{2 n+1}\left(C E\left(H_{2 n+1}(\cdot)\right) ; \omega_{k_{1}}, \cdots, \omega_{k_{2 n+1}}\right)
\end{aligned}
$$

and $\varphi_{2 n+1}\left(C E\left(H_{2 n+1}(\cdot)\right) ; \omega_{1}, \cdots, \omega_{2 n+1}\right)=\varphi_{2 n+1}\left(c_{3,0}(1,1,1)^{n} ; \omega_{1}, \cdots, \omega_{2 n+1}\right)$ can $\quad$ be $\quad$ obtained according to equations ( $9 \mathrm{a}-\mathrm{c}$ ). For example,

$$
\varphi_{3}\left(c_{3,0}(111) ; \omega_{1}, \omega_{2}, \omega_{3}\right)=\frac{1}{L_{3}\left(j \sum_{i=1}^{3} \omega_{i}\right)} \cdot \prod_{i=1}^{3}\left(j \omega_{i}\right) \cdot \prod_{i=1}^{3} H_{1}\left(j \omega_{i}\right)=\frac{\prod_{i=1}^{3}\left(j \omega_{i}\right)}{L_{3}\left(j \sum_{i=1}^{3} \omega_{i}\right)} \cdot \prod_{i=1}^{3} H_{1}\left(j \omega_{i}\right)
$$




$$
\begin{aligned}
& \varphi_{5}\left(c_{3,0}(111) c_{3,0}(111) ; \omega_{1}, \cdots, \omega_{5}\right) \\
& =f_{1}\left(c_{3,0}(111), 5 ; \omega_{1}, \cdots, \omega_{5}\right) \cdot \sum_{\begin{array}{l}
\text { all the } 3-\text { partitions } \\
\text { for } c_{3,0}(111)
\end{array}} \sum_{\begin{array}{l}
\text { all the different } \\
\text { permutations of }\{0,0,1\}
\end{array}}\left[f_{2 a}\left(s_{\bar{x}_{1}} \cdots s_{\bar{x}_{p}}\left(c_{3,0}(111)\right) ; \omega_{1} \cdots \omega_{5}\right)\right. \\
& \left.\cdot \prod_{i=1}^{3} \varphi_{n\left(s_{\bar{x}_{i}}\left(\bar{s} / c_{p, q}(\cdot)\right)\right)}\left(s_{\bar{x}_{i}}\left(c_{3,0}(111)\right) ; \omega_{l(\bar{X}(i)+1)} \cdots \omega_{l\left(\bar{X}(i)+n\left(s_{\bar{x}_{i}}\left(\bar{s} / c_{p, q}(\cdot)\right)\right)\right)}\right)\right] \\
& =f_{1}\left(c_{3,0}(111), 5 ; \omega_{1}, \cdots, \omega_{5}\right) \cdot\left(\begin{array}{l}
f_{2 a}\left(s_{0} s_{0} s_{1}\left(c_{3,0}(111)\right) ; \omega_{1} \cdots \omega_{5}\right) \varphi_{1}\left(1 ; \omega_{1}\right) \varphi_{1}\left(1 ; \omega_{2}\right) \varphi_{3}\left(c_{3,0}(111) ; \omega_{3} \cdots \omega_{5}\right) \\
+f_{2 a}\left(s_{0} s_{1} s_{0}\left(c_{3,0}(111)\right) ; \omega_{1} \cdots \omega_{5}\right) \varphi_{1}\left(1 ; \omega_{1}\right) \varphi_{3}\left(c_{3,0}(111) ; \omega_{2} \cdots \omega_{4}\right) \varphi_{1}\left(1 ; \omega_{5}\right) \\
+f_{2 a}\left(s_{1} s_{0} s_{0}\left(c_{3,0}(111)\right) ; \omega_{1} \cdots \omega_{5}\right) \varphi_{3}\left(c_{3,0}(111) ; \omega_{1} \cdots \omega_{3}\right) \varphi_{1}\left(1 ; \omega_{4}\right) \varphi_{1}\left(1 ; \omega_{5}\right)
\end{array}\right) \\
& =\frac{1}{L_{5}\left(j \sum_{i=1}^{5} \omega_{i}\right)} \cdot\left(\frac{\left(j \sum_{i=3}^{5} \omega_{i}\right) \prod_{i=1}^{5}\left(j \omega_{i}\right)}{L_{3}\left(j \sum_{i=3}^{5} \omega_{i}\right)}+\frac{\left(j \sum_{i=2}^{4} \omega_{i}\right) \prod_{i=1}^{5}\left(j \omega_{i}\right)}{L_{3}\left(j \sum_{i=2}^{4} \omega_{i}\right)}+\frac{\left(j \sum_{i=1}^{3} \omega_{i}\right) \prod_{i=1}^{5}\left(j \omega_{i}\right)}{L_{3}\left(j \sum_{i=1}^{3} \omega_{i}\right)}\right) \cdot \prod_{i=1}^{5} H_{1}\left(j \omega_{i}\right)
\end{aligned}
$$

where $\omega_{i} \in\{\Omega,-\Omega\}$, and so on. Substituting these results into Equations (18-19), the output spectrum is clearly a power series with respect to the parameter $c$. When there are more nonlinear terms, it is obvious that the computation process above can directly result in a straightforward multivariate power series with respect to these nonlinear parameters. To check the alternating phenomenon of the output spectrum, consider the following values for each linear parameter: $m=240, k_{0}=16000, B=296, F_{\mathrm{d}}=100$, and $\Omega=8.165$. Then it is obtained that

$$
\begin{aligned}
Y(j \Omega)= & \tilde{F}_{1}(\Omega)+c \tilde{F}_{3}(\Omega)+c^{2} \tilde{F}_{5}(\Omega)+\cdots \\
= & -j\left(\frac{F_{d}}{2}\right) H_{1}(j \Omega)+3\left(\frac{F_{d}}{2}\right)^{3} \frac{\Omega^{3} \mid H_{1}(j \Omega)^{2} H_{1}(j \Omega)}{L_{1}(j \Omega)} \\
& +3\left(\frac{F_{d}}{2}\right)^{5} \frac{\Omega^{5}\left|H_{1}(j \Omega)\right|^{4} H_{1}(j \Omega)}{L_{1}(j \Omega)}\left(\frac{j 6 \Omega}{L_{1}(j \Omega)}+\frac{j 3 \Omega}{L_{1}(j 3 \Omega)}+\frac{-j 3 \Omega}{L_{1}(-j \Omega)}\right)+\cdots \\
= & (-0.02068817126756+0.00000114704116 \mathrm{i}) \\
& +(5.982851578532449 \mathrm{e}-006-6.634300276113922 \mathrm{e}-010 \mathrm{i}) \mathrm{c} \\
+ & (-5.192417616715994 \mathrm{e}-009+3.323565122085705 \mathrm{e}-011 \mathrm{i}) \mathrm{c}^{2}+\ldots
\end{aligned}
$$

The series is alternating. In order to check the series further, computation of $\varphi_{2 n+1}\left(c_{3,0}(1,1,1)^{n} ; \omega_{1}, \cdots, \omega_{2 n+1}\right)$ can be carried out for higher orders. It can also be verified that the magnitude square of the output spectrum (20a) is still an alternating series, i.e.,

$$
|Y(j \Omega)|^{2}=(4.280004317115985 \mathrm{e}-004)-(2.475485177721052 \mathrm{e}-007) \mathrm{c}
$$




$$
+(2.506378395908398 \mathrm{e}-010) \mathrm{c}^{2}-\ldots
$$

As pointed in Theorem 1, it is easy to find a $c$ such that (20a-b) are convergent and their limits are decreased. From $(20 \mathrm{~b})$ and according to Theorem 1, it can be computed that $0.01671739<|Y(j \Omega)|<0.0192276<0.0206882$ for $c=600$. This can be verified by Figure 1 . Figure 1 is a result from simulation tests, and shows that the magnitude of the output spectrum is decreasing when $c$ is increasing. This property is of great significance in practical engineering systems for output suppression through structural characteristic design or feedback control.

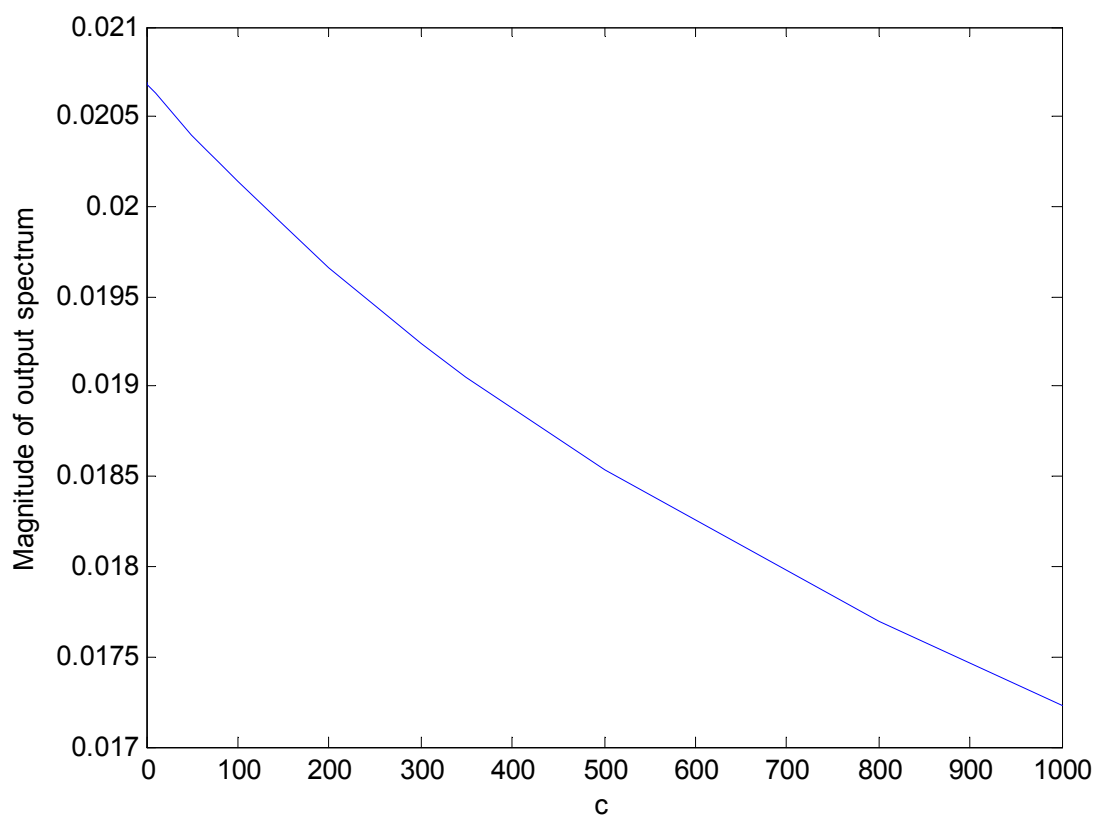

Figure 1. Magnitude of output spectrum

\section{Alternating conditions}

In this section, the conditions under which the output spectrum described by Equation (12) can be expressed into an alternating series with respect to any nonlinear parameter are studied. Suppose the system subjects to a harmonic input $u(t)=F_{d} \sin (\Omega t) \quad\left(F_{d}>0\right)$ and only the output nonlinearities (i.e., $c_{\mathrm{p}, 0}($.$) with p \geq 2$ ) are considered. For convenience, assume that there is only one nonlinear parameter $c_{p, 0}($.$) in model (2) and all the other$ nonlinear parameters are zero. The results for this case can be extended to the general one.

Under the assumptions above, it can be obtained from the parametric characteristic analysis in [6] as demonstrated in Example 2 and Equation (11b) that 


$$
\begin{aligned}
Y(j \Omega) & =Y_{1}(j \Omega)+Y_{p}(j \Omega)+\cdots+Y_{(p-1) n+1}(j \Omega)+\cdots \\
& =\tilde{F}_{1}(\Omega)+c_{p, 0}(\cdot) \tilde{F}_{p}(\Omega)+\cdots+c_{p, 0}(\cdot)^{n} \tilde{F}_{(p-1) n+1}(\Omega)+\cdots
\end{aligned}
$$

where $\omega_{k_{i}} \in\{ \pm \Omega\}, \quad \tilde{F}_{(p-1) n+1}(j \Omega)$ can be computed from $(11 \mathrm{~b})$, and $n$ is a positive integer. Noting that $F\left(\omega_{k_{l}}\right)=-j k_{l} F_{d}, k_{l}= \pm 1, \omega_{k_{l}}=k_{l} \Omega$, and $l=1, \cdots, n$ in $(11 \mathrm{~b})$,

$$
\begin{aligned}
\tilde{F}_{(p-1) n+1}(j \Omega) & =\frac{1}{2^{(p-1) n+1}} \\
& \sum_{\omega_{k_{1}}+\cdots+\omega_{k_{(p-1) n+1}}=\Omega} \varphi_{(p-1) n+1}\left(c_{p, 0}(\cdot)^{n} ; \omega_{k_{1}}, \cdots, \omega_{k_{(p-1) n+1}}\right) \cdot\left(-j F_{d}\right)^{(p-1) n+1} \cdot k_{1} k_{2} \cdots k_{(p-1) n+1}
\end{aligned}
$$

If $p$ is an odd integer, then $(p-1) n+1$ is also an odd integer. Thus there should be $(p-1) n / 2$ frequency variables being $-\Omega$ and $(p-1) n / 2+1$ frequency variables being $\Omega$ such that $\omega_{k_{1}}+\cdots+\omega_{k_{(p-1) n+1}}=\Omega$. In this case,

$$
\left(-j F_{d}\right)^{(p-1) n+1} \cdot k_{1} k_{2} \cdots k_{(p-1) n+1}=(-1) \cdot j \cdot\left(j^{2}\right)^{(p-1) n / 2} \cdot\left(F_{d}\right)^{(p-1) n+1} \cdot(-1)^{(p-1) n / 2}=-j\left(F_{d}\right)^{(p-1) n+1}
$$

If $p$ is an even integer, then $(p-1) n+1$ is an odd integer for $n=2 k(k=1,2,3, \ldots)$ and an even integer for $n=2 k-1(k=1,2,3, \ldots)$. When $n$ is an odd integer, $\omega_{k_{1}}+\cdots+\omega_{k_{(p-1) n+1}} \neq \Omega$ for $\omega_{k_{l}} \in\{ \pm \Omega\}$. This gives that $\tilde{F}_{(p-1) n+1}(j \Omega)=0$. When $n$ is an even integer, $(p-1) n+1$ is an odd integer. In this case, it is similar to that $p$ is an odd integer. Therefore, for $n>0$

$$
\widetilde{F}_{(p-1) n+1}(j \Omega)= \begin{cases}-j\left(\frac{F_{d}}{2}\right)^{(p-1) n+1} \sum_{\omega_{k_{1}}+\cdots+\omega_{k(p-1) n+1}=\Omega} \varphi_{(p-1) n+1}\left(c_{p, 0}(\cdot)^{n} ; \omega_{k_{1}}, \cdots, \omega_{k_{(p-1) n+1}}\right) & \text { if p is odd or n is even }(21 \mathrm{c}) \\ 0 & \text { else }\end{cases}
$$

From Equations (21a-c) it is obvious that the property of the new mapping $\varphi_{(p-1) n+1}\left(c_{p, 0}(\cdot)^{n} ; \omega_{k_{1}}, \cdots, \omega_{k_{(p-1) n+1}}\right)$ plays a key role in the series. To develop the alternating conditions for series (21a), the following results can be obtained.

Lemma 1. That $\varphi_{(p-1) n+1}\left(c_{p, 0}(\cdot)^{n} ; \omega_{k_{1}}, \cdots, \omega_{k_{(p-1) n+1}}\right)$ is symmetric or asymmetric has no influence on $\tilde{F}_{(p-1) n+1}(j \Omega)$.

Lemma 1 is obvious since $\sum_{\omega_{k_{1}}+\cdots+\omega_{k(p-1) n+1}=\Omega}(\cdot)$ includes all the possible permutations of $\left(\omega_{k_{1}}, \cdots, \omega_{k_{2 n+1}}\right)$. Although there are many choices to obtain the asymmetric $\varphi_{(p-1) n+1}\left(c_{p, 0}(\cdot)^{n} ; \omega_{k_{1}}, \cdots, \omega_{k_{(p-1) n+1}}\right)$ which may be different at different permutation $\left(\omega_{k_{1}}, \cdots, \omega_{k_{(p-1) n+1}}\right)$, they have no effect on the analysis of $\widetilde{F}_{(p-1) n+1}(j \Omega)$.

The following lemma is straightforward.

Lemma 2. For $v_{1}, v_{2}, v \in \mathbb{C}$, suppose $\operatorname{sgn}_{c}\left(v_{1}\right)=-\operatorname{sgn}_{c}\left(v_{2}\right)$. If $\operatorname{Re}(v) \operatorname{Im}(v)=0$, then $\operatorname{sgn}_{c}\left(v_{1} v\right)=-\operatorname{sgn}_{c}\left(v_{2} v\right)$. If $\operatorname{Re}(v) \operatorname{Im}(v)=0$ and $v \neq 0$, then $\operatorname{sgn}_{c}\left(v_{1} / v\right)=-\operatorname{sgn}_{c}\left(v_{2} / v\right) \square$. 
Theorem 2. The output spectrum in (21a-c) is an alternating series with respect to parameter $c_{\mathrm{p}, 0}\left(\mathrm{k}_{1}, \mathrm{k}_{2}, \ldots, \mathrm{k}_{\mathrm{p}}\right)$ satisfying $c_{p, 0}()>$.0 and $p=2 r+1$ for $\mathrm{r}=1,2,3, \ldots$

(1) if and only if

$$
\begin{aligned}
& \operatorname{sgn}_{c}\left(\sum_{\omega_{k_{1}}+\cdots+\omega_{k_{(p-1) n+1}}=\Omega}(-1)^{n-1} \varphi_{(p-1) n+1}\left(c_{p, 0}(\cdot)^{n} ; \omega_{l(1)} \cdots \omega_{l((p-1) n+1)}\right)\right)=\text { const , i.e., }
\end{aligned}
$$

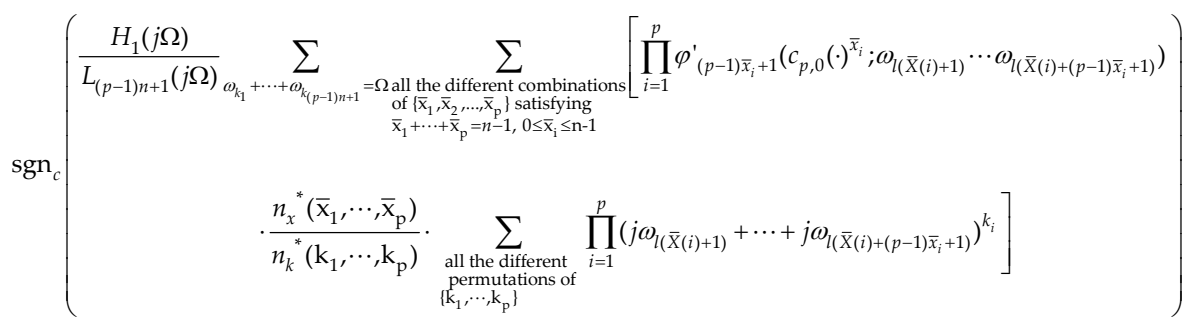

where const is a two-dimensional constant vector whose elements are $+1,0$ or -1 ;

$$
\begin{aligned}
& \varphi_{(p-1) n+1}^{\prime}\left(c_{p, 0}(\cdot)^{n} ; \omega_{l(1)} \cdots \omega_{l((p-1) n+1)}\right) \\
& =\frac{-1}{L_{(p-1) n+1}\left(j \omega_{l(1)}+\cdots+j \omega_{l((p-1) n+1)}\right)} \cdot \sum_{\begin{array}{l}
\text { all the different combinations } \\
\text { of }\left\{\bar{x}_{1}, \bar{x}_{2}, \ldots, \bar{p}_{\mathrm{p}}\right\} \text { satisfying } \\
\bar{x}_{1}+\cdots+\bar{x}_{\mathrm{p}}=n-1,0 \leq \overline{\mathrm{x}}_{i} \leq n-1
\end{array}}\left[\prod_{i=1}^{p} \varphi_{(p-1) \bar{x}_{i}+1}^{\prime}\left(c_{p, 0}(\cdot)^{\bar{x}_{i}} ; \omega_{l(\bar{X}(i)+1)} \cdots \omega_{l\left(\bar{X}(i)+(p-1) \bar{x}_{i}+1\right)}\right)\right. \\
& \left.\cdot \frac{n_{x}^{*}\left(\overline{\mathrm{x}}_{1}, \cdots, \overline{\mathrm{x}}_{\mathrm{p}}\right)}{n_{k}^{*}\left(\mathrm{k}_{1}, \cdots, \mathrm{k}_{\mathrm{p}}\right)} \cdot \sum_{\substack{\text { all the different } \\
\text { permutations of } \\
\left\{\mathrm{k}_{1}, \cdots, \mathrm{k}_{\mathrm{p}}\right\}}} \prod_{i=1}^{p}\left(j \omega_{l(\bar{X}(i)+1)}+\cdots+j \omega_{l\left(\bar{X}(i)+(p-1) \bar{x}_{i}+1\right)}\right)^{k_{i}}\right]
\end{aligned}
$$

the termination is $\varphi_{1}^{\prime}\left(1 ; \omega_{i}\right)=1 ; n_{k}^{*}\left(k_{1}, \cdots, k_{p}\right)=\frac{p !}{n_{1} ! n_{2} ! \cdots n_{e} !}, n_{1}+\ldots+n_{\mathrm{e}}=p, e$ is the number of distinct differentials $k_{\mathrm{i}}$ appearing in the combination, $n_{\mathrm{i}}$ is the number of repetitions of $k_{\mathrm{i}}$, and a similar definition holds for $n_{x}^{*}\left(\bar{x}_{1}, \cdots, \bar{x}_{p}\right)$.

(2) or if $k_{1}=k_{2}=\ldots=k_{\mathrm{p}}=k$ in $c_{p, 0}(),. \operatorname{Re}\left(\frac{H_{1}(j \Omega)}{L_{(p-1) n+1}(j \Omega)}\right) \operatorname{Im}\left(\frac{H_{1}(j \Omega)}{L_{(p-1) n+1}(j \Omega)}\right)=0$, and

$$
\operatorname{sgn}_{c}\left(\begin{array}{c}
\sum_{\begin{array}{l}
\sum_{k_{1}}+\cdots+\omega_{k_{(p-1) n+1}}=\Omega \text { all the different combinations } \\
\text { of }\left\{\overline{\mathrm{x}}_{1}, \overline{\mathrm{x}}_{2}, \ldots, \bar{x}_{\mathrm{p}}\right\} \text { satisfying } \\
\overline{\mathrm{x}}_{1}+\cdots+\overline{\mathrm{x}}_{\mathrm{p}}=n-1,0 \leq \overline{\mathrm{x}}_{\mathrm{i}} \leq \mathrm{n}-1
\end{array}}^{p}\left[n_{x}^{*}\left(\overline{\mathrm{x}}_{1}, \cdots, \overline{\mathrm{x}}_{\mathrm{p}}\right)\right. \\
\left.\cdot \prod_{i=1}^{p} \varphi_{(p-1) \bar{x}_{i}+1}^{\prime \prime}\left(c_{p, 0}(\cdot)^{\bar{x}_{i}} ; \omega_{l(\overline{\mathrm{X}}(i)+1)} \cdots \omega_{l\left(\bar{X}(i)+(p-1) \bar{x}_{i}+1\right)}\right)\right]
\end{array}\right)=\text { const }
$$


where if $\bar{x}_{i}=0, \varphi_{(p-1) \bar{x}_{i}+1}^{\prime \prime}\left(c_{p, 0}(\cdot)^{\bar{x}_{i}} ; \omega_{l(\bar{X}(i)+1)} \cdots \omega_{l\left(\bar{X}(i)+(p-1) \bar{x}_{i}+1\right)}\right)=1$, otherwise,

$$
\begin{aligned}
& \varphi_{(p-1) \bar{x}_{i}+1}^{\prime \prime}\left(c_{p, 0}(\cdot)^{\bar{x}_{i}} ; \omega_{l(\bar{X}(i)+1)} \cdots \omega_{l\left(\bar{X}(i)+(p-1) \bar{x}_{i}+1\right)}\right) \\
& =\frac{\left(j \omega_{l(\bar{X}(i)+1)}+\cdots+j \omega_{l\left(\bar{X}(i)+(p-1) \bar{x}_{i}+1\right)}\right)^{k}}{-L_{(p-1) \bar{x}_{i}+1}\left(j \omega_{l(\bar{X}(i)+1)}+\cdots+j \omega_{l\left(\bar{X}(i)+(p-1) \bar{x}_{i}+1\right)}\right)} . \\
& \sum_{\text {all the different combinations }} n_{x}^{*}\left(\mathrm{x}_{1}, \cdots, \mathrm{x}_{\mathrm{p}}\right) \cdot \prod_{j=1}^{p} \varphi_{(p-1) x_{j}+1}^{\prime \prime}\left(c_{p, 0}(\cdot)^{x_{j}} ; \omega_{l\left(\bar{X}^{\prime}(j)+1\right)} \cdots \omega_{l\left(\bar{X}^{\prime}(j)+(p-1) x_{j}+1\right)}\right) \\
& \text { of }\left\{x_{1}, x_{2}, \ldots, x_{p}\right\} \text { satisfying } \\
& \mathrm{x}_{1}+\cdots+\mathrm{x}_{\mathrm{p}}=\bar{x}_{i}-1,0 \leq \mathrm{x}_{\mathrm{j}} \leq \bar{x}_{i}-1
\end{aligned}
$$

The recursive terminal of $\varphi_{(p-1) \bar{x}_{i}+1}^{\prime \prime}\left(c_{p, 0}(\cdot)^{\bar{x}_{i}} ; \omega_{l(\bar{X}(i)+1)} \cdots \omega_{l\left(\bar{X}(i)+(p-1) \bar{x}_{i}+1\right)}\right)$ is $\bar{x}_{i}=1$.

Proof. See Appendix D.

Theorem 2 provides a sufficient and necessary condition for the output spectrum series (21ac) to be an alternating series with respect to a nonlinear parameter $c_{\mathrm{p}, 0}\left(\mathrm{k}_{1}, \mathrm{k}_{2}, \ldots, \mathrm{k}_{\mathrm{p}}\right)$ satisfying $c_{\mathrm{p}, 0}($. $)>0$ and $p=2 r+1$ for $\mathrm{r}=1,2,3, \ldots$. Similar results can also be established for any other nonlinear parameters. Regarding nonlinear parameter $c_{\mathrm{p}, 0}\left(\mathrm{k}_{1}, \mathrm{k}_{2}, \ldots, \mathrm{k}_{\mathrm{p}}\right)$ satisfying $c_{\mathrm{p}, 0}()>$.0 and $p=2 r$ for $\mathrm{r}=1,2,3, \ldots . .$, it can be obtained from $(21 \mathrm{a}-\mathrm{c})$ that

$$
Y(j \Omega)=\tilde{F}_{1}(\Omega)+c_{p, 0}(\cdot)^{2} \tilde{F}_{2(p-1)+1}(\Omega)+\cdots+c_{p, 0}(\cdot)^{2 n} \tilde{F}_{2(p-1) n+1}(\Omega)+\cdots
$$

$\tilde{F}_{2(p-1) n+1}(\Omega)$ for $\mathrm{n}=1,2,3, \ldots$ should be alternating so that $Y(j \Omega)$ is alternating. This yields that

$$
\begin{aligned}
& \operatorname{sgn}_{c}\left(\sum_{\omega_{k_{1}}+\cdots+\omega_{k_{2(p-1) n+1}=\Omega}} \varphi_{2(p-1) n+1}\left(c_{p, 0}(\cdot)^{2 n} ; \omega_{l(1)} \cdots \omega_{l(2(p-1) n+1)}\right)\right) \\
& \quad=-\operatorname{sgn}_{c}\left(\sum_{\omega_{k_{1}}+\cdots+\omega_{k_{2(p-1)(n+1)+1}}=\Omega} \varphi_{2(p-1)(n+1)+1}\left(c_{p, 0}(\cdot)^{2(n+1)} ; \omega_{l(1)} \cdots \omega_{l(2(p-1)(n+1)+1)}\right)\right)
\end{aligned}
$$

Clearly, this is different from the conditions in Theorem 2. It may be more difficult for the output spectrum to be alternating with respect to $c_{\mathrm{p}, 0}($.) $>0$ with $p=2 r$ (even degree) than with respect to $c_{\mathrm{p}, 0}()>$.0 with $p=2 r+1$ (odd degree).

Note that Equation (21a) is based on the assumption that there is only nonlinear parameter $c_{\mathrm{p}, 0}($.$) and all the other nonlinear parameters are zero. If the effects from the other nonlinear$ parameters are considered, Equation (21a) can be written as

$$
Y(j \Omega)=\tilde{F}_{1}^{\prime}(\Omega)+c_{p, 0}(\cdot) \tilde{F}_{p}^{\prime}(\Omega)+\cdots+c_{p, 0}(\cdot)^{n} \tilde{F}_{(p-1) n+1}^{\prime}(\Omega)+\cdots
$$

where 


$$
\tilde{F}_{(p-1) n+1}^{\prime}(\Omega)=\tilde{F}_{(p-1) n+1}(\Omega)+\delta_{(p-1) n+1}\left(\Omega ; C_{p^{\prime}, q^{\prime}} \backslash c_{p, 0}(.)\right)
$$

$C_{p^{\prime}, q^{\prime}}$ includes all the nonlinear parameters in the system. Based on the parametric characteristic analysis in [6] and the new mapping function $\varphi_{n}\left(C E\left(H_{n}(\cdot)\right) ; \omega_{1}, \cdots, \omega_{n}\right)$ defined in [7], (24b) can be determined easily. For example, suppose $p$ is an odd integer larger than 1 , then $\tilde{F}_{(p-1) n+1}(j \Omega)$ is given in (21c), and $\delta_{(p-1) n+1}\left(\Omega ; C_{p^{\prime}, q^{\prime}} \backslash c_{p, 0}().\right)$ can be computed as

$$
\begin{aligned}
& \delta_{(p-1) n+1}\left(\Omega ; C_{p^{\prime}, q^{\prime}} \backslash C_{p, 0}(.)\right)=\sum_{\begin{array}{l}
\text { all the monomails consisting of the parameters in } C_{p^{\prime}, q^{\prime}} \backslash c_{p, 0}(\cdot) \\
\text { satisfying np+ }+\left(\mathrm{p}_{\mathrm{i}}^{\prime}+\mathrm{q}_{\mathrm{i}}^{\prime}\right) \text { is odd and less than } \mathrm{N}
\end{array}}\left[-j\left(\frac{F_{d}}{2}\right)^{n\left(c_{p, 0}{ }^{n} s(\cdot)\right)}\right. \\
& \left.\sum_{\omega_{k_{1}}+\cdots+\omega_{k_{n\left(c_{p, 0}\right.} n_{s(\cdot))}}=\Omega} \varphi_{n\left(c_{p, 0}{ }^{n} s(\cdot)\right)}\left(c_{p, 0}{ }^{n} s\left(C_{p^{\prime}, q^{\prime}} \backslash c_{p, 0}(\cdot)\right) ; \omega_{k_{1}} \cdots \omega_{k\left(c_{p, 0}{ }^{n} s(\cdot)\right)}\right)\right]
\end{aligned}
$$

where $s\left(C_{p^{\prime}, q^{\prime}} \backslash c_{p, 0}(\cdot)\right)$ denotes a monomial consisting of some parameters in $C_{p^{\prime}, q^{\prime}} \backslash c_{p, 0}(\cdot)$.

It is obvious that if (21a) is an alternating series, then (24a) can still be alternating under a proper design of the other nonlinear parameters (for example the other parameters are sufficiently small). Moreover, from the discussions above, it can be seen that whether the system output spectrum is an alternating series or not with respect to a specific nonlinear parameter is greatly dependent on the system linear parameters.

Example 3. To demonstrate the theoretical results above, consider again model (15) in Example 2. Let $u(t)=F_{d} \sin (\Omega t) \quad\left(F_{d}>0\right)$. The output spectrum at frequency $\Omega$ is given in (18-19). From Lemma 3 in Appendix D, it can be derived for this case that

$$
\begin{gathered}
\varphi_{2 n+1}\left(c_{3,0}(\cdot)^{n} ; \omega_{l(1)} \cdots \omega_{l(2 n+1)}\right)=\frac{(-1)^{n-1} \prod_{i=1}^{2 n+1}\left[\left(j \omega_{l(i)}\right)^{k} H_{1}\left(j \omega_{l(i)}\right)\right]}{L_{2 n+1}\left(j \omega_{l(1)}+\cdots+j \omega_{l(2 n+1)}\right)} \\
\cdot \sum_{\substack{\text { all the different combinations } \\
\text { of }\left\{\bar{x}_{1}, \overline{\mathrm{x}}_{2}, \overline{\mathrm{x}}_{3}\right\} \text { satisfying } \\
\overline{\mathrm{x}}_{1}+\overline{\mathrm{x}}_{2}+\overline{\mathrm{x}}_{3}=n-1,0 \leq \bar{x}_{\mathrm{i}} \leq \mathrm{n}-1}} n_{x}^{*}\left(\overline{\mathrm{x}}_{1}, \overline{\mathrm{x}}_{2}, \overline{\mathrm{x}}_{3}\right) \cdot \prod_{i=1}^{3} \varphi_{2 \bar{x}_{i}+1}^{\prime \prime}\left(c_{3,0}(\cdot)^{\bar{x}_{i}} ; \omega_{l(\overline{\mathrm{X}}(i)+1)} \cdots \omega_{l\left(\overline{\mathrm{X}}(i)+2 \bar{x}_{i}+1\right)}\right)
\end{gathered}
$$

where, if $\bar{x}_{i}=0, \varphi_{(p-1) \bar{x}_{i}+1}^{\prime \prime}\left(c_{p, 0}(\cdot)^{\bar{x}_{i}} ; \omega_{l(\bar{X}(i)+1)} \cdots \omega_{l\left(\bar{X}(i)+(p-1) \bar{x}_{i}+1\right)}\right)=1$, otherwise,

$$
\begin{aligned}
& \varphi_{2 \bar{x}_{i}+1}^{\prime \prime}\left(c_{3,0}(\cdot)^{\bar{x}_{i}} ; \omega_{l(\bar{X}(i)+1)} \cdots \omega_{l\left(\bar{X}(i)+2 \bar{x}_{i}+1\right)}\right) \\
& =\frac{\left(j \omega_{l(\bar{X}(i)+1)}+\cdots+j \omega_{l\left(\bar{X}(i)+2 \bar{x}_{i}+1\right)}\right)^{k}}{-L_{2 \bar{x}_{i}+1}\left(j \omega_{l(\bar{X}(i)+1)}+\cdots+j \omega_{l\left(\bar{X}(i)+2 \bar{x}_{i}+1\right)}\right)} \cdot \\
& \sum_{\substack{\text { all the different combinations } \\
\text { of }\left\{\mathrm{x}_{1}, \mathrm{x}_{2}, \mathrm{x}_{3}\right\} \text { satisfying } \\
\mathrm{x}_{1}+\mathrm{x}_{2}+\mathrm{x}_{3}=\bar{x}_{i}-1,0 \leq \mathrm{x}_{\mathrm{j}} \leq \bar{x}_{i}-1}} n_{x}^{*}\left(\mathrm{x}_{1}, \mathrm{x}_{2}, \mathrm{x}_{3}\right) \cdot \prod_{j=1}^{3} \varphi_{2 x_{j}+1}^{\prime \prime}\left(c_{3,0}(\cdot)^{x_{j}} ; \omega_{l\left(\bar{X}^{\prime}(j)+1\right)} \cdots \omega_{l\left(\bar{X}^{\prime}(j)+2 x_{j}+1\right)}\right)
\end{aligned}
$$


Note that the terminal condition for $(25 b)$ is at $\bar{x}_{i}=1$, i.e.,

$$
\left.\varphi_{2 \bar{x}_{i}+1}^{\prime \prime}\left(c_{3,0}(\cdot)^{\bar{x}_{i}} ; \omega_{l(\bar{X}(i)+1)} \cdots \omega_{l\left(\bar{X}(i)+2 \bar{x}_{i}+1\right)}\right)\right|_{\bar{x}_{i}=1}=\varphi_{3}^{\prime \prime}\left(c_{3,0}(\cdot) ; \omega_{l(1)} \cdots \omega_{l(3)}\right)=\frac{\left(j \omega_{l(1)}+\cdots+j \omega_{l(3)}\right)^{k}}{-L_{3}\left(j \omega_{l(1)}+\cdots+j \omega_{l(3)}\right)}
$$

Therefore, from (25a-c) it can be easily shown that $\varphi_{2 n+1}\left(c_{3,0}(\cdot)^{n} ; \omega_{1} \cdots \omega_{2 n+1}\right)$ can be written as

$$
\begin{aligned}
& \varphi_{2 n+1}\left(c_{3,0}(\cdot)^{n} ; \omega_{1} \cdots \omega_{2 n+1}\right) \\
& =\frac{(-1)^{n-1} \prod_{i=1}^{2 n+1} j \omega_{i} H_{1}\left(j \omega_{i}\right)}{L_{2 n+1}\left(j \omega_{1}+\cdots+j \omega_{2 n+1}\right)} \cdot \sum_{\begin{array}{l}
\text { all the combination }\left(x_{1}, x_{2}, \ldots, x_{n-1}\right) \\
\text { satisfying } x_{i} \in\{j+11 \leq j \leq n-1\} \\
x_{1} \geq x_{2} \geq . \geq x_{n-1} \text { and } \\
="=\text { happens only if } x_{\mathrm{i}}+x_{i+1} \leq 2 n-2
\end{array}} r_{X}\left(\mathrm{x}_{1}, \mathrm{x}_{2}, \cdots, \mathrm{x}_{\mathrm{n}-1)}\right) \prod_{i=1}^{n-1} \frac{j \omega_{l(1)}+\cdots+j \omega_{l\left(x_{i}\right)}}{-L_{x_{i}}\left(j \omega_{l(1)}+\cdots+j \omega_{l\left(x_{i}\right)}\right)}
\end{aligned}
$$

where $r_{X}\left(\mathrm{x}_{1}, \mathrm{x}_{2}, \ldots, \mathrm{x}_{\mathrm{n}-1}\right)$ is a positive integer which can be explicitly determined by (25ab) and represents the number of all the involved combinations which have the same $\prod_{i=1}^{n-1} \frac{j \omega_{l(1)}+\cdots+j \omega_{l\left(x_{i}\right)}}{-L_{x_{i}}\left(j \omega_{l(1)}+\cdots+j \omega_{l\left(x_{i}\right)}\right)}$. Therefore, according to the sufficient condition in Theorem 2, it can be seen from (26) that the output spectrum (18) is an alternating series only if the following two conditions hold:

(a1) $\quad \operatorname{Re}\left(\frac{H_{1}(j \Omega)}{L_{2 n+1}(j \Omega)}\right) \operatorname{Im}\left(\frac{H_{1}(j \Omega)}{L_{2 n+1}(j \Omega)}\right)=0$

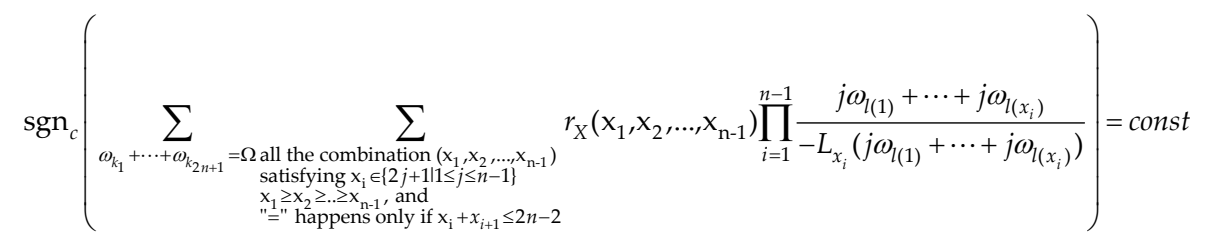

Suppose $\Omega=\sqrt{\frac{k_{0}}{m}}$ which is a natural resonance frequency of model (15). It can be derived that

$$
\begin{gathered}
L_{2 n+1}(j \Omega)=-\sum_{k_{1}=0}^{K} c_{1,0}\left(k_{1}\right)(j \Omega)^{k_{1}}=-\left(m(j \Omega)^{2}+B(j \Omega)+k_{0}\right)=-j B \Omega \\
H_{1}(j \Omega)=\frac{-1}{L_{1}(j \Omega)}=\frac{1}{j B \Omega}
\end{gathered}
$$

It is obvious that condition (a1) above is satisfied if $\Omega=\sqrt{\frac{k_{0}}{m}}$. Considering condition (a2), it can be derived that 


$$
\frac{j \omega_{l(1)}+\cdots+j \omega_{l\left(x_{i}\right)}}{-L_{x_{i}}\left(j \omega_{l(1)}+\cdots+j \omega_{l\left(x_{i}\right)}\right)}=\frac{j \varepsilon\left(x_{i}\right) \Omega}{-L_{x_{i}}\left(j \varepsilon\left(x_{i}\right) \Omega\right)}
$$

where $\varepsilon\left(x_{i}\right) \in\{ \pm(2 j+1) \mid 0 \leq j \leq\lceil n+1\rceil\}$, and $\lceil n+1\rceil$ denotes the odd integer not larger than $\mathrm{n}+1$. Especially, when $\varepsilon\left(x_{i}\right)= \pm 1$, it yields that

$$
\frac{j \omega_{l(1)}+\cdots+j \omega_{l\left(x_{i}\right)}}{-L_{x_{i}}\left(j \omega_{l(1)}+\cdots+j \omega_{l\left(x_{i}\right)}\right)}=\frac{ \pm j \Omega}{-L_{x_{i}}( \pm j \Omega)}=\frac{ \pm j \Omega}{ \pm j B \Omega}=\frac{1}{B}
$$

when $\left|\varepsilon\left(x_{i}\right)\right|>1$,

$$
\begin{aligned}
& \frac{j \omega_{l(1)}+\cdots+j \omega_{l\left(x_{i}\right)}}{-L_{x_{i}}\left(j \omega_{l(1)}+\cdots+j \omega_{l\left(x_{i}\right)}\right)}=\frac{j \varepsilon\left(x_{i}\right) \Omega}{-L_{x_{i}}\left(j \varepsilon\left(x_{i}\right) \Omega\right)}=\frac{j \varepsilon\left(x_{i}\right) \Omega}{m\left(j \varepsilon\left(x_{i}\right) \Omega\right)^{2}+B\left(j \varepsilon\left(x_{i}\right) \Omega\right)+k_{0}} \\
& =\frac{j \varepsilon\left(x_{i}\right) \Omega}{\left(1-\varepsilon\left(x_{i}\right)^{2}\right) k_{0}+j \varepsilon\left(x_{i}\right) \Omega B}=\frac{1}{B+j\left(\varepsilon\left(x_{i}\right)-\frac{1}{\varepsilon\left(x_{i}\right)}\right) \sqrt{k_{0} m}}
\end{aligned}
$$

If $B<\sqrt{k_{0} m}$, then it gives

$$
\frac{j \omega_{l(1)}+\cdots+j \omega_{l\left(x_{i}\right)}}{-L_{x_{i}}\left(j \omega_{l(1)}+\cdots+j \omega_{l\left(x_{i}\right)}\right)} \approx \frac{1}{j\left(\varepsilon\left(x_{i}\right)-\frac{1}{\varepsilon\left(x_{i}\right)}\right) \sqrt{k_{0} m}}
$$

Note that in all the combinations involved in the summation operator in (26) or condition (a2), i.e.,

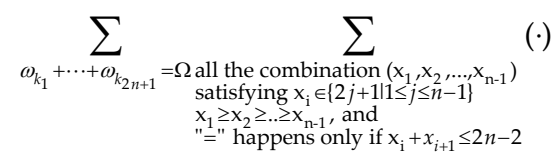

There always exists a combination such that

$$
\prod_{i=1}^{n-1} \frac{j \omega_{l(1)}+\cdots+j \omega_{l\left(x_{i}\right)}}{-L_{x_{i}}\left(j \omega_{l(1)}+\cdots+j \omega_{l\left(x_{i}\right)}\right)}=\frac{1}{B^{n-1}}
$$

Note that (27b) holds for both $\varepsilon\left(x_{i}\right)= \pm 1$, thus there is no combination such that

$$
\prod_{i=1}^{n-1} \frac{j \omega_{l(1)}+\cdots+j \omega_{l\left(x_{i}\right)}}{-L_{x_{i}}\left(j \omega_{l(1)}+\cdots+j \omega_{l\left(x_{i}\right)}\right)}=-\frac{1}{B^{n-1}}
$$

Also noting that $B<<\sqrt{k_{0} m}$, all these show that

$$
\max _{\substack{\text { all the involved } \\ \text { combinations }}} \mid\left(\prod_{i=1}^{n-1} \frac{j \omega_{l(1)}+\cdots+j \omega_{l\left(x_{i}\right)}}{-L_{x_{i}}\left(j \omega_{l(1)}+\cdots+j \omega_{l\left(x_{i}\right)}\right)} \mid\right)=\frac{1}{B^{n-1}}
$$


which happens in the combination where (28) holds.

Because there are $n+1$ frequency variables to be $+\Omega$ and $n$ frequency variables to be $-\Omega$ such that $\omega_{1}+\cdots+\omega_{2 n+1}=\Omega$ in (18-19), there are more combinations where $\varepsilon\left(x_{i}\right)>0$ that is $\left(\varepsilon\left(x_{i}\right)-\frac{1}{\varepsilon\left(x_{i}\right)}\right) \sqrt{k_{0} m}>0$ in $(27 \mathrm{c}-\mathrm{d})$. Thus there are more combinations where $\operatorname{Im}($ $\left.\frac{j \omega_{l(1)}+\cdots+j \omega_{l\left(x_{i}\right)}}{-L_{x_{i}}\left(j \omega_{l(1)}+\cdots+j \omega_{l\left(x_{i}\right)}\right)}\right)$ is negative. Using $(27 \mathrm{~b})$ and $(27 \mathrm{~d})$, it can be shown under the condition that $B<<\sqrt{k_{0} m}$,

$$
\left.\max _{\begin{array}{c}
\text { all the involved } \\
\text { combinations }
\end{array}}\left|\operatorname{Im}\left(\prod_{i=1}^{n-1} \frac{j \omega_{l(1)}+\cdots+j \omega_{l\left(x_{i}\right)}}{-L_{x_{i}}\left(j \omega_{l(1)}+\cdots+j \omega_{l\left(x_{i}\right)}\right)}\right)\right|\right)\left.\approx \frac{1}{B^{n-2}\left(\varepsilon\left(x_{i}\right)-\frac{1}{\varepsilon\left(x_{i}\right)}\right) \sqrt{k_{0} m}}\right|_{\varepsilon\left(x_{i}\right)=3}=\frac{1}{2.7 B^{n-2} \sqrt{k_{0} m}}
$$

This happens in the combinations where the argument of $\prod_{i=1}^{n-1} \frac{j \omega_{l(1)}+\cdots+j \omega_{l\left(x_{i}\right)}}{-L_{x_{i}}\left(j \omega_{l(1)}+\cdots+j \omega_{l\left(x_{i}\right)}\right)}$ is either $-90^{\circ}$ or $+90^{\circ}$. Note that there are more cases in which the arguments are $-90^{\circ}$. If the argument is $-180^{\circ}$, the absolute value of the corresponding imaginary part will be not more than

$$
\left.\max _{\substack{\text { the combination } \\ \text { whose argument is } \\-180^{0}}}\left(\operatorname{Im}\left(\prod_{i=1}^{n-1} \frac{j \omega_{l(1)}+\cdots+j \omega_{l\left(x_{i}\right)}}{-L_{x_{i}}\left(j \omega_{l(1)}+\cdots+j \omega_{l\left(x_{i}\right)}\right)}\right) \mid\right) \approx \frac{1}{B^{n-4}\left(\varepsilon\left(x_{i}\right)-\frac{1}{\varepsilon\left(x_{i}\right)}\right)^{3}{\sqrt{k_{0} m}}^{3}}\right|_{\varepsilon\left(x_{i}\right)=3}=\frac{1}{2.7^{3} B^{n-4}{\sqrt{k_{0} m}}^{3}}
$$

which is much less than $\frac{1}{2.7 B^{n-2} \sqrt{k_{0} m}}$.

Therefore, if $B$ is sufficiently smaller than $\sqrt{k_{0} m}$, the following two inequalities can hold for $n>1$

$$
\begin{aligned}
& \operatorname{Re}\left(\sum_{\substack{\text { all the combination } \\
\text { satisfying } \mathrm{x}_{\mathrm{i}} \in\{2 j+1 \mid 1 \leq j \leq 1 \leq n-1\} \\
\mathrm{x}_{1} \geq \mathrm{x}_{2} \geq . . \geq \mathrm{x}_{\mathrm{n}-1}, \text { and } \\
\text { "=" happens only if } \mathrm{x}_{\mathrm{i}}+x_{i+1} \leq 2 n-2}} r_{X}\left(\mathrm{x}_{1}, \mathrm{x}_{2}, \ldots, \mathrm{x}_{\mathrm{n}-1}\right) \prod_{i=1}^{n-1} \frac{j \omega_{l(1)}+\cdots+j \omega_{l\left(x_{i}\right)}}{-L_{x_{i}}\left(j \omega_{l(1)}+\cdots+j \omega_{l\left(x_{i}\right)}\right)}\right)>0 \\
& \operatorname{Im}\left(\sum_{\substack{\text { all the combination }\left(x_{1}, x_{2}, \ldots, x_{n-1}\right) \\
\text { satisfying } \mathrm{x}_{\mathrm{i}} \in\{2 j+1 \mid 1 \leq j \leq n-1\} \\
\mathrm{x}_{1} \geq \mathrm{x}_{2} \geq . .2 \mathrm{x}_{\mathrm{n}-1} \text { and } \\
\text { "=" happens only if } \mathrm{x}_{\mathrm{i}}+x_{i+1} \leq 2 n-2}} r_{X}\left(\mathrm{x}_{1}, \mathrm{x}_{2}, \ldots, \mathrm{x}_{\mathrm{n}-1}\right) \prod_{i=1}^{n-1} \frac{j \omega_{l(1)}+\cdots+j \omega_{l\left(x_{i}\right)}}{-L_{x_{i}}\left(j \omega_{l(1)}+\cdots+j \omega_{l\left(x_{i}\right)}\right)}\right)<0
\end{aligned}
$$

That is, condition (a2) holds for $n>1$ under $B<<\sqrt{k_{0} m}$ and $\Omega=\sqrt{\frac{k_{0}}{m}}$. Hence, (18) is an alternating series if the following two conditions hold:

(b1) $B$ is sufficiently smaller than $\sqrt{k_{0} m}$, 
(b2) The input frequency is $\Omega=\sqrt{\frac{k_{0}}{m}}$.

In Example 2, note that $\Omega=\sqrt{\frac{k_{0}}{m}} \approx 8.165, \mathrm{~B}=296<<\sqrt{k_{0} m}=1959.592$. These are consistent with the theoretical results established above. As it has been checked numerically in Example 2 that (18) is an alternating series, the theoretical results above are well verified by the real system.

Therefore, it can be seen that, at the driving frequency the system output spectrum (subject to a cubic nonlinear damping) can be designed to be an alternating series by properly designing system parameters (see conditions (b1-b2) above) and therefore can be suppressed as shown in Example 2 by properly choosing a value for the nonlinear parameter $c$. This result clearly demonstrate the mechanism for the nonlinear effect of the cubic nonlinear damping in the frequency domain.

More simulation studies about the properties of the cubic nonlinear damping can be referred to the simulation results in [8], where the effects of the cubic nonlinear damping are studied in details and compared with a linear damping. The case study here theoretically shows for the first time why and when these nonlinear effects happen and what the underlying mechanism is.

Based on the discussions in Examples 2-3, it can be concluded that, the results of this study provide a new systematic method for the analysis and design of the nonlinear effect for a class of nonlinearities in the frequency domain.

\section{Conclusions}

Nonlinear influence on system output spectrum is investigated in this study from a novel perspective based on Volterra series expansion in the frequency domain. For a class of system nonlinearities, it is shown that system output spectrum can be expanded into an alternating series with respect to nonlinear parameters of the model under certain conditions and this alternating series has some interesting properties for engineering practices. Although there may be several existing methods such as perturbation analysis that can achieve similar objectives for some simple cases in practice, this study proposes a novel viewpoint on the nonlinear effect (i.e., alternating series) and on the analysis of nonlinear effect (i.e., the GFRFs-based) for a class of nonlinearities in the frequency domain. As some important properties of a linear system (e.g. stability) are determined by the positions of the poles of its transfer function, the fact of alternating series should be a natural characteristic of some important nonlinear effects for nonlinear systems in the frequency domain. This study provides some fundamental results for characterizing and understanding of nonlinear effects in the frequency domain from this novel viewpoint. The GFRFs-based analysis provides a useful technique for the analysis of nonlinear systems which is just similar to the transfer function based analysis for linear systems. The method demonstrated in this paper has been used for the analysis and design of nonlinear damping 
systems. Further study will focus on more detailed design and analysis methods based on these results for practical systems.

\section{Author details}

Xingiian Jing

Department of Mechanical Engineering, Hong Kong Polytechnic University, Hung Hom, Kowloon, Hong Kong

\section{Acknowledgement}

The author gratefully acknowledges the support of a GRF project of Hong Kong RGC (Ref 517810), the Department General Research Funds and Internal Competitive Research Grants of Hong Kong Polytechnic University for this work.

\section{Appendix}

\section{Appendix A: Nomenclature}

$c_{p, q}\left(k_{1}, \cdots, k_{p+q}\right)$ - A model parameter in the NDE model, $k_{\mathrm{i}}$ is the order of the derivative, $p$ represents the order of the involved output nonlinearity, $q$ is the order of the involved input nonlinearity, and $p+q$ is the nonlinear degree of the parameter.

$H_{n}\left(j \omega_{1}, \cdots, j \omega_{n}\right)$ - The nth-order GFRF

$C_{p, q}=\left[c_{p, q}(0, \cdots, 0), c_{p, q}(0, \cdots, 1), \cdots, c_{p, q}(\underbrace{K, \cdots, K}_{p+q=m})\right]$ - A parameter vector consisting of all the nonlinear parameters of the form ..

$C E($.$) - The coefficient extraction operator$

$C E\left(H_{n}\left(j \omega_{1}, \cdots, j \omega_{n}\right)\right)$ - The parametric characteristics of the nth-order GFRF

$f_{n}\left(j \omega_{1}, \cdots, j \omega_{n}\right)$ - The correlative function of $C E\left(H_{n}\left(j \omega_{1}, \cdots, j \omega_{n}\right)\right)$

$\otimes$ - The reduced Kronecker product defined in the CE operator

$\oplus$ - The reduced vectorized summation defined in the CE operator

$\underset{(*)}{\otimes(\cdot)}$ and $\underset{(*)}{\oplus}(\cdot)$ - The multiplication and addition by the reduced Kronecker product " $\otimes$ " and vectorized sum “ $\oplus$ " of the terms in (.) satisfying $\left({ }^{*}\right)$, respectively

$$
\begin{aligned}
& \bigotimes_{i=1}^{k} C_{p, q}=C_{p, q} \otimes \cdots \otimes C_{p, q} \text { - can be simply written as } C_{p, q}^{k} . \\
& c_{p_{0}, q_{0}}(\cdot) c_{p_{1}, q_{1}}(\cdot) \cdots c_{p_{k}, q_{k}}(\cdot) \text { - A monomial consisting of nonlinear parameters }
\end{aligned}
$$


$s_{x_{1}} s_{x_{2}} \cdots s_{x_{p}}$ - A p -partition of a monomial $c_{p_{0}, q_{0}}(\cdot) c_{p_{1}, q_{1}}(\cdot) \cdots c_{p_{k}, q_{k}}(\cdot)$

$s_{x_{i}}$ - A monomial of $x_{\mathrm{i}}$ parameters of $\left\{c_{p_{0}, q_{0}}(\cdot), \cdots, c_{p_{k}, q_{k}}(\cdot)\right\}$ of the involved monomial, $0 \leq x_{i} \leq k$, and $\mathrm{s}_{0}=1$

$\varphi_{n}: S_{C}(n) \rightarrow S_{f}(n)$ - A new mapping function from the parametric characteristics to the correlative functions, $S_{C}(n)$ is the set of all the monomials in the parametric characteristics and $S_{f}(n)$ is the set of all the involved correlative functions in the nth order GFRF.

$n\left(s_{x}(\bar{s})\right)$ - The order of the GFRF where the monomial $s_{x}(\bar{s})$ is generated

\section{Appendix B: The Coefficient Extraction (CE) operator $[6,7,22,23]$}

Consider a series

$$
H_{C F}=c_{1} f_{1}+c_{2} f_{2}+\cdots+c_{\sigma} f_{\sigma} \in \Xi
$$

where the coefficients $c_{i}(i=1, \ldots, \sigma)$ are different monomial functions in a set $P_{c}$ of some parameters in a set $C_{s}$ which takes values in $\mathbb{C}, f_{i}$ for $i=1, \ldots, n$ are some complex valued scalar functions in a set $P_{f}$ which are independent of the parameters in $C_{s}, \Xi$ denotes all the finite order series with coefficients in $P_{c}$ timing some functions in $P_{f}, C=\left[c_{1}, C_{2}, \ldots, c_{\sigma}\right]$, and $F=[$ $\left.f_{1}, f_{2}, \ldots, f_{\sigma}\right]^{\mathrm{T}}$. Define a Coefficient Extraction operator $C E: \Xi \rightarrow \mathbb{C}^{\sigma}$ for this series such that

$$
C E\left(H_{C F}\right)=\left[c_{1}, c_{2}, \cdots, c_{\sigma}\right]=C \in \mathbb{C}^{\sigma}
$$

where $\mathbb{C}^{\sigma}$ is the $\sigma$-dimensional complex valued vector space. This operator has the following properties, also acting as operation rules:

1. Reduced vectorized sum “ $\oplus ”$.

$$
C E\left(H_{C_{1} F_{1}}+H_{C_{2} F_{2}}\right)=C E\left(H_{C_{1} F_{1}}\right) \oplus C E\left(H_{C_{2} F_{2}}\right)=C_{1} \oplus C_{2}=\left[C_{1}, C_{2}^{\prime}\right], \quad C_{2}^{\prime}=\operatorname{VEC}\left(\bar{C}_{2}-\bar{C}_{1} \cap \bar{C}_{2}\right),
$$
where $\bar{C}_{1}=\left\{C_{1}(i)|1 \leq i \leq| C_{1} \mid\right\}, \bar{C}_{2}=\left\{C_{2}(i)|1 \leq i \leq| C_{2} \mid\right\}, V E C($.$) is a vector consisting of all$ the elements in set (.). $C_{2}^{\prime}$ is a vector including all the elements in $C_{2}$ except the same elements as those in $\mathrm{C}_{1}$.

2. Reduced Kronecker product “ $\otimes "$.

$$
C E\left(H_{C_{1} F_{1}} \cdot H_{C_{2} F_{2}}\right)=C E\left(H_{C_{1} F_{1}}\right) \otimes C E\left(H_{C_{2} F_{2}}\right)=C_{1} \otimes C_{2}=\operatorname{VEC}\left\{C_{3}(i) \mid \begin{array}{l}
C_{3}=\left[C_{1}(1) C_{2}, \cdots, C_{1}\left(\left|C_{1}\right|\right) C_{2}\right] \\
1 \leq i \leq\left|C_{3}\right|
\end{array}\right\}
$$

which implies that there are no repetitive elements in $C_{1} \otimes C_{2}$.

3. Invariant. (a) $C E\left(\alpha \cdot H_{C F}\right)=C E\left(H_{C F}\right) \quad \forall \alpha \in \mathbb{C}$ but is not a parameter of interest; (b)

$$
C E\left(H_{C F_{1}}+H_{C F_{2}}\right)=C E\left(H_{C\left(F_{1}+F_{2}\right)}\right)=C
$$


4. Unitary. If $H_{C F}$ is not a function of $c_{i}$ for $i=1 \ldots n, C E\left(H_{C F}\right)=1$.

When there is a unitary 1 in $C E\left(H_{C F}\right)$, there is a nonzero constant term in the corresponding series $H_{C F}$ which has no relation with the coefficients $c_{i}$ (for $i=1 \ldots n$ ). In addition, if $H_{C F}=0$, then $\mathrm{CE}\left(\mathrm{H}_{\mathrm{CF}}\right)=0$.

5. Inverse. $C E^{-1}(C)=H_{C F}$.

6. $C E\left(H_{C_{1} F_{1}}\right) \approx C E\left(H_{C_{2} F_{2}}\right)$ if the elements of $C_{1}$ are the same as those of $C_{2}$, where " $\approx$ " means equivalence, i.e., both series are in fact the same result considering the order of $c_{i} f_{i}$ in the series has no effect on the value of a function series $H_{с F}$. This further implies that the $C E$ operator is also commutative and associative, for instance, $C E\left(H_{C_{1} F_{1}}+H_{C_{2} F_{2}}\right)=C_{1} \oplus C_{2} \approx C E\left(H_{C_{2} F_{2}}+H_{C_{1} F_{1}}\right)=C_{2} \oplus C_{1}$. Hence, the results by the CE operator with respect to the same purpose may be different but all correspond to the same function series and are thus equivalent.

7. Separable and interested parameters only. A parameter in a series can only be extracted if the parameter is interested and the series is separable with respect to this interested parameter. Thus the operation result is different for different purposes.

\section{Appendix C: Proof of theorem 1}

(1) $Y(j \omega)$ is convergent if and only if $\operatorname{Re}(Y(j \omega))$ and $\operatorname{Im}(Y(j \omega))$ are both convergent. Since $Y(j \omega)$ is an alternating series, $\operatorname{Re}(Y(j \omega))$ and $\operatorname{Im}(Y(j \omega))$ are both alternating from Definition

1. Then according to [3], $\operatorname{Re}(Y(j \omega))$ is convergent if $\left|\operatorname{Re}\left(c^{i} F_{i}(j \omega)\right)\right|>\left|\operatorname{Re}\left(c^{i+1} F_{i+1}(j \omega)\right)\right|$ and $\lim _{i \rightarrow \infty}\left|\operatorname{Re}\left(c^{i} F_{i}(j \omega)\right)\right|=0$. Therefore, if there exists $T>0$ such that $\left|\operatorname{Re}\left(c^{i} F_{i}(j \omega)\right)\right|>\left|\operatorname{Re}\left(c^{i+1} F_{i+1}(j \omega)\right)\right|$ for $i>T$ and $\lim _{i \rightarrow \infty}\left|\operatorname{Re}\left(c^{i} F_{i}(j \omega)\right)\right|=0$, the alternating series $\operatorname{Re}(Y(j \omega))$ is also convergent. Now since there exist $T>0$ and $R>0$ such that $-\frac{\operatorname{Re}\left(F_{i}(j \omega)\right)}{\operatorname{Re}\left(F_{i+1}(j \omega)\right)}>R$ for $i>T$ and note $c<R$, it can be obtained that for $i>T$

$$
-\frac{\operatorname{Re}\left(c^{i+1} F_{i+1}(j \omega)\right)}{\operatorname{Re}\left(c^{i} F_{i}(j \omega)\right)}=-\frac{\operatorname{Re}\left(c F_{i+1}(j \omega)\right)}{\operatorname{Re}\left(F_{i}(j \omega)\right)}=\left|\frac{\operatorname{Re}\left(c F_{i+1}(j \omega)\right)}{\operatorname{Re}\left(F_{i}(j \omega)\right)}\right|<\frac{c}{R}<1
$$

i.e., $\left|\operatorname{Re}\left(c^{i} F_{i}(j \omega)\right)\right|>\left|\operatorname{Re}\left(c^{i+1} F_{i+1}(j \omega)\right)\right|$ for $i>T$ and $c<R$. Moreover, it can also be obtained that for $n>0$

$$
\left|\operatorname{Re}\left(F_{T+n}(j \omega)\right)\right|<\frac{1}{R^{n}}\left|\operatorname{Re}\left(F_{T}(j \omega)\right)\right|
$$

It further yields that

$$
\left|\operatorname{Re}\left(c^{T+n} F_{T+n}(j \omega)\right)\right|<\left(\frac{c}{R}\right)^{n} c^{T}\left|\operatorname{Re}\left(F_{T}(j \omega)\right)\right|
$$


That is, $\lim _{n \rightarrow \infty}\left|\operatorname{Re}\left(c^{T+n} F_{T+n}(j \omega)\right)\right|=0$. Therefore, $\operatorname{Re}(Y(j \omega))$ is convergent. Similarly, it can be proved that $\operatorname{Im}(Y(j \omega))$ is convergent. This proves that $Y(j \omega)$ is convergent. According to [3], the truncation errors for the convergent alternating series $\operatorname{Re}(Y(j \omega))$ and $\operatorname{Im}(Y(j \omega))$ are bounded by

$$
\left|o_{R}(\rho)\right| \leq c^{\rho+1}\left|\operatorname{Re}\left(F_{\rho+1}(j \omega)\right)\right| \text { and }\left|o_{I}(\rho)\right| \leq c^{\rho+1}\left|\operatorname{Im}\left(F_{\rho+1}(j \omega)\right)\right|
$$

Therefore, the truncation error for the series $Y(j \omega)$ is

$$
|o(\rho)|=\sqrt{o_{R}(\rho)^{2}+o_{I}(\rho)^{2}} \leq c^{\rho+1}\left|F_{\rho+1}(j \omega)\right|
$$

Since $\operatorname{Re}(Y(j \omega))$ and $\operatorname{Im}(Y(j \omega))$ are both alternating series and the absolute value of each term in $\operatorname{Re}(Y(j \omega))$ and $\operatorname{Im}(Y(j \omega))$ are monotone decreasing, i.e., $\left|\operatorname{Re}\left(c^{i} F_{i}(j \omega)\right)\right|>\left|\operatorname{Re}\left(c^{i+1} F_{i+1}(j \omega)\right)\right|$ and $\left|\operatorname{Im}\left(c^{i} F_{i}(j \omega)\right)\right|>\left|\operatorname{Im}\left(c^{i+1} F_{i+1}(j \omega)\right)\right|$ for $i>T$, then it can be shown for $\operatorname{Re}(Y(j \omega))$ and $\operatorname{Im}(Y(j \omega))$ that for $n \geq 0$

$$
\begin{aligned}
& \left|\operatorname{Re}\left(Y(j \omega)_{1 \rightarrow T+1}\right)\right|<\cdots<\left|\operatorname{Re}\left(Y(j \omega)_{1 \rightarrow T+2 n+1}\right)\right|<|\operatorname{Re}(Y(j \omega))|<\left|\operatorname{Re}\left(Y(j \omega)_{1 \rightarrow T+2 n}\right)\right|<\cdots<\left|\operatorname{Re}\left(Y(j \omega)_{1 \rightarrow T}\right)\right| \\
& \left|\operatorname{Im}\left(Y(j \omega)_{1 \rightarrow T+1}\right)\right|<\cdots<\left|\operatorname{Im}\left(Y(j \omega)_{1 \rightarrow T+2 n+1}\right)\right|<|\operatorname{Im}(Y(j \omega))|<\left|\operatorname{Im}\left(Y(j \omega)_{1 \rightarrow T+2 n}\right)\right|<\cdots<\left|\operatorname{Im}\left(Y(j \omega)_{1 \rightarrow T}\right)\right|
\end{aligned}
$$

Therefore, $\left|Y(j \omega)_{1 \rightarrow T+1}\right|<\cdots<\left|Y(j \omega)_{1 \rightarrow T+2 n+1}\right|<|Y(j \omega)|<\left|Y(j \omega)_{1 \rightarrow T+2 n}\right|<\cdots<\left|Y(j \omega)_{1 \rightarrow T}\right|$.

(2)

$$
\begin{aligned}
|Y(j \omega)|^{2} & =Y(j \omega) Y(-j \omega) \\
& =\left(F_{0}(j \omega)+c F_{1}(j \omega)+c^{2} F_{2}(j \omega)+\cdots\right)\left(F_{0}(-j \omega)+c F_{1}(-j \omega)+c^{2} F_{2}(-j \omega)+\cdots\right) \\
& =\sum_{n=0,1,2, \ldots} c^{n} \sum_{i=0}^{n} F_{i}(j \omega) F_{n-i}(-j \omega)
\end{aligned}
$$

It can be verified that the $(2 k)$ th terms in the series are positive and the $(2 k+1)$ th terms are negative for $k=0,1,2, \ldots$. Moreover, it is not difficult to obtain that it needs only the real parts of the terms in $Y(j \omega)$ to be alternating for $|Y(j \omega)|^{2}=Y(j \omega) Y(-j \omega)$ to be alternating. (3)

$$
\begin{aligned}
\frac{\partial|Y(j \omega)|}{\partial c} & =\frac{1}{2|Y(j \omega)|} \frac{\partial|Y(j \omega)|^{2}}{\partial c} \\
& =\frac{1}{2|Y(j \omega)|}\left\{\operatorname{Re}\left(F_{0}(j \omega) F_{1}(-j \omega)\right)+c \sum_{n=1,2, \ldots} n c^{n-1} \sum_{i=0}^{n} F_{i}(j \omega) F_{n-i}(-j \omega)\right\}
\end{aligned}
$$

Since $\operatorname{Re}\left(F_{0}(j \omega) F_{1}(-j \omega)\right)<0$, there must exist $\bar{c}>0$ such that $\frac{\partial|Y(j \omega)|}{\partial c}<0$ for $0<c<\bar{c}$. This completes the proof. 


\section{Appendix D: Proof of theorem 2}

In order to prove Theorem 2, the following lemma is needed, which provides a fundamental technique for the derivation of the main results in Theorem 2 by exploiting the recursive nature of $\varphi_{n(\bar{s})}\left(c_{p, 0}(\cdot)^{n} ; \omega_{l(1)} \cdots \omega_{l(n(\bar{s}))}\right)$.

Lemma 3. Consider a nonlinear parameter denoted by $c_{\mathrm{p}, \mathrm{q}}\left(k_{1}, k_{2}, \ldots, k_{\mathrm{p}+\mathrm{q}}\right)$.

(1) If $p \geq 2$ and $q=0$, then

$$
\begin{aligned}
& \varphi_{n(\bar{s})}\left(c_{p, 0}(\cdot)^{n} ; \omega_{l(1)} \cdots \omega_{l(n(\bar{s}))}\right)=\varphi_{(p-1) n+1}\left(c_{p, 0}(\cdot)^{n} ; \omega_{l(1)} \cdots \omega_{l((p-1) n+1)}\right) \\
& =\frac{(-1)^{n-1} \prod_{i=1}^{(p-1) n+1} H_{1}\left(j \omega_{l(i)}\right)}{L_{(p-1) n+1}\left(j \omega_{l(1)}+\cdots+j \omega_{l((p-1) n+1)}\right)} \cdot \sum_{\begin{array}{l}
\text { all the different combinations } \\
\text { of }\left\{\overline{\mathrm{x}}_{1}, \overline{\mathrm{x}}_{2}, \ldots, \bar{x}_{\mathrm{p}}\right\} \text { satisfying } \\
\overline{\mathrm{x}}_{1}+\cdots+\overline{\mathrm{x}}_{\mathrm{p}}=n-1,0 \leq \overline{\mathrm{x}}_{\mathrm{i}} \leq \mathrm{n}-1
\end{array}}\left[\prod_{i=1}^{p} \varphi_{(p-1) \bar{x}_{i}+1}^{\prime}\left(c_{p, 0}(\cdot)^{\bar{x}_{i}} ; \omega_{l(\bar{X}(i)+1)} \cdots \omega_{l\left(\bar{X}(i)+(p-1) \bar{x}_{i}+1\right)}\right)\right. \\
& \left.\cdot \frac{n_{x}^{*}\left(\overline{\mathrm{x}}_{1}, \cdots, \overline{\mathrm{x}}_{\mathrm{p}}\right)}{n_{k}{ }^{*}\left(\mathrm{k}_{1}, \cdots, \mathrm{k}_{\mathrm{p}}\right)} \cdot \sum_{\substack{\text { all the different } \\
\text { permutations of } \\
\left\{\mathrm{k}_{1}, \cdots, \mathrm{k}_{\mathrm{p}}\right\}}} \prod_{i=1}^{p}\left(j \omega_{l(\bar{X}(i)+1)}+\cdots+j \omega_{l\left(\bar{X}(i)+(p-1) \bar{x}_{i}+1\right)}\right)^{k_{i}}\right]
\end{aligned}
$$

where,

$$
\begin{gathered}
\varphi_{(p-1) n+1}^{\prime}\left(c_{p, 0}(\cdot)^{n} ; \omega_{l(1)} \cdots \omega_{l((p-1) n+1)}\right) \\
=\frac{-1}{L_{(p-1) n+1}\left(j \omega_{l(1)}+\cdots+j \omega_{l((p-1) n+1)}\right)} \cdot \sum_{\begin{array}{c}
\text { all the different combinations } \\
\text { of }\left\{\bar{x}_{1}, \bar{x}_{2}, \cdots, \bar{x}_{\mathrm{p}}\right\} \text { satisfying } \\
\overline{\mathrm{x}}_{1}+\cdots+\overline{\mathrm{x}}_{\mathrm{p}}=n-1,0 \leq \overline{\mathrm{x}}_{\mathrm{i}} \leq \mathrm{n}-1
\end{array}}\left[\prod_{i=1}^{p} \varphi_{(p-1) \bar{x}_{i}+1}^{\prime}\left(c_{p, 0}(\cdot)^{\bar{x}_{i}} ; \omega_{l(\bar{X}(i)+1)} \cdots \omega_{l\left(\bar{X}(i)+(p-1) \bar{x}_{i}+1\right)}\right)\right. \\
\left.\cdot \frac{n_{x}^{*}\left(\overline{\mathrm{x}}_{1}, \cdots, \overline{\mathrm{x}}_{\mathrm{p}}\right)}{n_{k}^{*}\left(\mathrm{k}_{1}, \cdots, \mathrm{k}_{\mathrm{p}}\right)} \cdot \sum_{\substack{\text { all the different } \\
\text { permutations of } \\
\left\{\mathrm{k}_{1}, \cdots, \mathrm{k}_{\mathrm{p}}\right\}}} \prod_{i=1}^{p}\left(j \omega_{l(\bar{X}(i)+1)}+\cdots+j \omega_{l\left(\bar{X}(i)+(p-1) \bar{x}_{i}+1\right)}\right)^{k_{i}}\right]
\end{gathered}
$$

the termination is $\varphi_{1}^{\prime}\left(1 ; \omega_{i}\right)=1 . n_{k}^{*}\left(k_{1}, \cdots, k_{p}\right)=\frac{p !}{n_{1} ! n_{2} ! \cdots n_{e} !}, n_{1}+\ldots+n_{\mathrm{e}}=p, e$ is the number of distinct differentials $k_{\mathrm{i}}$ appearing in the combination, $n_{\mathrm{i}}$ is the number of repetitions of $k_{\mathrm{i}}$, and a similar definition holds for $n_{x}^{*}\left(\bar{x}_{1}, \cdots, \bar{x}_{p}\right)$.

(2) If $p \geq 2, q=0$ and $k_{1}=k_{2}=\ldots=k_{\mathrm{p}}=k$, then

$$
\begin{aligned}
& \varphi_{(p-1) n+1}\left(c_{p, 0}(\cdot)^{n} ; \omega_{l(1)} \cdots \omega_{l((p-1) n+1)}\right) \\
& =\frac{(-1)^{n-1} \prod_{i=1}^{(p-1) n+1}\left[\left(j \omega_{l(i)}\right)^{k} H_{1}\left(j \omega_{l(i)}\right)\right]}{L_{(p-1) n+1}\left(j \omega_{l(1)}+\cdots+j \omega_{l((p-1) n+1)}\right)} \\
& \cdot \sum_{\substack{\text { all the different combinations } \\
\text { of }\left\{\overline{\mathrm{x}}_{1}, \overline{\mathrm{x}}_{2}, \ldots, \overline{\mathrm{x}}_{\mathrm{p}}\right\} \text { satisfying } \\
\overline{\mathrm{x}}_{1}+\cdots+\overline{\mathrm{x}}_{\mathrm{p}}=n-1,0 \leq \overline{\mathrm{x}}_{\mathrm{i}} \leq \mathrm{n}-1}} n_{x}^{*}\left(\overline{\mathrm{x}}_{1}, \cdots, \overline{\mathrm{x}}_{\mathrm{p}}\right) \cdot \prod_{i=1}^{p} \varphi_{(p-1) \bar{x}_{i}+1}^{\prime \prime}\left(c_{p, 0}(\cdot)^{\bar{x}_{i}} ; \omega_{l(\bar{X}(i)+1)} \cdots \omega_{l\left(\bar{X}(i)+(p-1) \bar{x}_{i}+1\right)}\right)
\end{aligned}
$$


where, if $\bar{x}_{i}=0, \varphi_{(p-1) \bar{x}_{i}+1}^{\prime \prime}\left(c_{p, 0}(\cdot)^{\bar{x}_{i}} ; \omega_{l(\bar{X}(i)+1)} \cdots \omega_{l\left(\bar{X}(i)+(p-1) \bar{x}_{i}+1\right)}\right)=1$, otherwise,

$$
\begin{aligned}
& \varphi_{(p-1) \bar{x}_{i}+1}^{\prime \prime}\left(c_{p, 0}(\cdot)^{\bar{x}_{i}} ; \omega_{l(\bar{X}(i)+1)} \cdots \omega_{l\left(\bar{X}(i)+(p-1) \bar{x}_{i}+1\right)}\right) \\
& =\frac{\left(j \omega_{l(\bar{X}(i)+1)}+\cdots+j \omega_{l\left(\bar{X}(i)+(p-1) \bar{x}_{i}+1\right)}\right)^{k}}{-L_{(p-1) \bar{x}_{i}+1}\left(j \omega_{l(\bar{X}(i)+1)}+\cdots+j \omega_{l\left(\bar{X}(i)+(p-1) \bar{x}_{i}+1\right)}\right)} . \\
& \sum_{\text {all the different combinations }} n_{x}{ }^{*}\left(\mathrm{x}_{1}, \cdots, \mathrm{x}_{\mathrm{p}}\right) \cdot \prod_{j=1}^{p} \varphi_{(p-1) x_{j}+1}^{\prime \prime}\left(c_{p, 0}(\cdot)^{x_{j}} ; \omega_{l\left(\bar{X}^{\prime}(j)+1\right)} \cdots \omega_{l\left(\bar{X}^{\prime}(j)+(p-1) x_{j}+1\right)}\right) \\
& \text { of }\left\{x_{1}, x_{2}, \ldots, x_{p}\right\} \text { satisfying } \\
& \mathrm{x}_{1}+\cdots+\mathrm{x}_{\mathrm{p}}=\bar{x}_{i}-1,0 \leq \mathrm{x}_{\mathrm{j}} \leq \bar{x}_{i}-1
\end{aligned}
$$

The recursive terminal of $\varphi_{(p-1) \bar{x}_{i}+1}^{\prime \prime}\left(c_{p, 0}(\cdot)^{\bar{x}_{i}} ; \omega_{l(\bar{X}(i)+1)} \cdots \omega_{l\left(\bar{X}(i)+(p-1) \bar{x}_{i}+1\right)}\right)$ is $\bar{x}_{i}=1$.

Proof of Lemma 3.

$$
\begin{aligned}
& \varphi_{n(\bar{s})}\left(c_{p, 0}(\cdot)^{n} ; \omega_{l(1)} \cdots \omega_{l(n(\bar{s}))}\right)=\varphi_{(p-1) n+1}\left(c_{p, 0}(\cdot) c_{p, 0}(\cdot) \cdots c_{p, 0}(\cdot) ; \omega_{l(1)} \cdots \omega_{(l(p-1) n+1)}\right)
\end{aligned}
$$

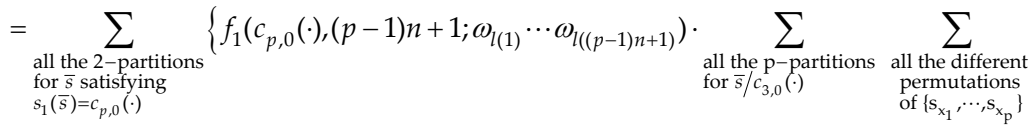

$$
\begin{aligned}
& \left.\left[f_{2 a}\left(s_{\bar{x}_{1}} \cdots s_{\bar{x}_{p}}\left(c_{p 0}(\cdot)^{n-1}\right) ; \omega_{l(1)} \cdots \omega_{l(n(\bar{s}))}\right) \cdot \prod_{i=1}^{p} \varphi_{n\left(s_{\bar{x}_{i}}\left(c_{p, 0}(\cdot)^{n-1}\right)\right)}\left(s_{\bar{x}_{i}}\left(c_{p, 0}(\cdot)^{n-1}\right) ; \omega_{l(\bar{X}(i)+1)} \cdots \omega_{l\left(\bar{X}(i)+n\left(s_{\bar{x}_{i}}\left(c_{p, 0}(\cdot)^{n-1}\right)\right)\right)}\right)\right]\right\}
\end{aligned}
$$

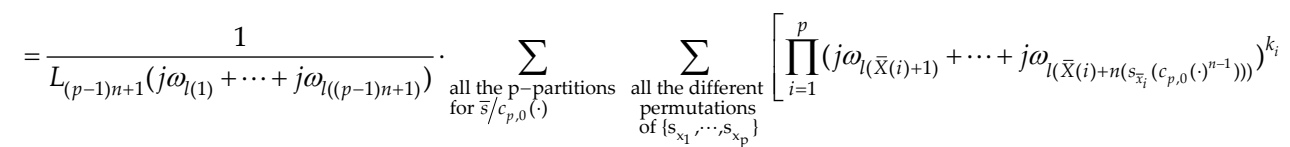

$$
\begin{aligned}
& \left.\cdot \prod_{i=1}^{p} \varphi_{n\left(\bar{s}_{\overline{x_{i}}}\left(c_{p, 0}(\cdot)^{n-1}\right)\right)}\left(s_{\bar{x}_{i}}\left(c_{p, 0}(\cdot)^{n-1}\right) ; \omega_{l(\bar{X}(i)+1)} \cdots \omega_{l\left(\bar{X}(i)+n\left(s_{\bar{x}_{i}}\left(c_{p, 0}(\cdot)^{n-1}\right)\right)\right)}\right)\right]
\end{aligned}
$$

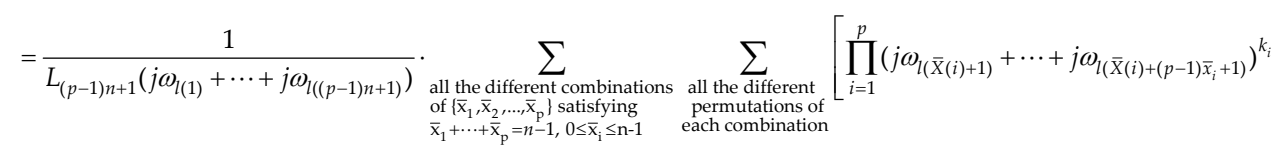

$$
\begin{aligned}
& \left.\cdot \prod_{i=1}^{p} \varphi_{(p-1) \bar{x}_{i}+1}\left(c_{p, 0}(\cdot)^{\bar{x}_{i}} ; \omega_{l(\bar{X}(i)+1)} \cdots \omega_{l\left(\bar{X}(i)+(p-1) \bar{x}_{i}+1\right)}\right)\right]
\end{aligned}
$$

Note that different permutations in each combination have no difference to $\prod_{i=1}^{p} \varphi_{(p-1) \bar{x}_{i}+1}\left(c_{p, 0}(\cdot)^{\bar{x}_{i}} ; \omega_{l(\bar{X}(i)+1)} \cdots \omega_{l\left(\bar{X}(i)+(p-1) \bar{x}_{i}+1\right)}\right)$, thus $\varphi_{(p-1) n+1}\left(c_{p, 0}(\cdot)^{n} ; \omega_{1} \cdots \omega_{(p-1) n+1}\right)$ can be written as 


$$
\begin{aligned}
& \varphi_{(p-1) n+1}\left(c_{p, 0}(\cdot)^{n} ; \omega_{1} \cdots \omega_{(p-1) n+1}\right) \\
& =\frac{1}{L_{(p-1) n+1}\left(j \omega_{l(1)}+\cdots+j \omega_{l((p-1) n+1)}\right)} \cdot \sum_{\begin{array}{l}
\text { all the different combinations } \\
\text { of }\left\{\bar{x}_{1}, \overline{\mathrm{x}}_{2}, \ldots, \overline{\mathrm{x}}_{\mathrm{p}}\right\} \text { satisfying } \\
\overline{\mathrm{x}}_{1}+\cdots+\overline{\mathrm{x}}_{3}=n-1,0 \leq \overline{\mathrm{x}}_{\mathrm{i}} \leq \mathrm{n}-1
\end{array}}^{p} \prod_{(p-1) \bar{x}_{i}+1}\left(c_{p, 0}(\cdot)^{\bar{x}_{i}} ; \omega_{l(\bar{X}(i)+1)} \cdots \omega_{l\left(\bar{X}(i)+(p-1) \bar{x}_{i}+1\right)}\right) \\
& \sum_{\substack{\text { all the different } \\
\text { permutations of } \\
\text { each combination }}} \prod_{i=1}^{p}\left(j \omega_{l(\bar{X}(i)+1)}+\cdots+j \omega_{l\left(\bar{X}(i)+(p-1) \bar{x}_{i}+1\right)}\right)^{k_{i}} \\
& =\frac{1}{L_{(p-1) n+1}\left(j \omega_{l(1)}+\cdots+j \omega_{l((p-1) n+1)}\right)} \cdot \sum_{\begin{array}{l}
\text { all the different combinations } \\
\text { of }\left\{\overline{\mathrm{x}}_{1}, \overline{\mathrm{x}}_{2}, \ldots, \overline{\mathrm{x}}_{\mathrm{p}}\right\} \text { satisfying } \\
\overline{\mathrm{x}}_{1}+\cdots+\overline{\mathrm{x}}_{\mathrm{p}}=n-1,0 \leq \overline{\mathrm{x}}_{\mathrm{i}} \leq \mathrm{n}-1
\end{array}}^{p} \prod_{(p-1) \bar{x}_{i}+1}\left(c_{p, 0}(\cdot)^{\bar{x}_{i}} ; \omega_{l(\overline{\mathrm{X}}(i)+1)} \cdots \omega_{l\left(\bar{X}(i)+(p-1) \bar{x}_{i}+1\right)}\right)
\end{aligned}
$$

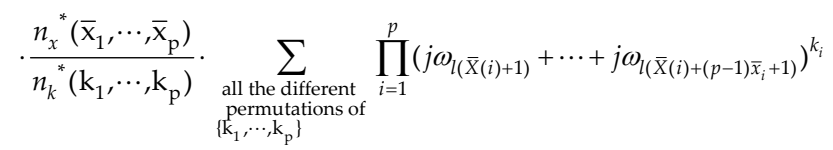

$n_{x}^{*}\left(\bar{x}_{1}, \cdots, \bar{x}_{p}\right)$ and $n_{k}^{*}\left(k_{1}, \cdots, k_{p}\right)$ are the numbers of the corresponding combinations involved, which can be obtained from the combination theory and can also be referred to [14]. Inspection of the recursion in the equation above, it can be seen that there are $(p-1) n+1$ $H_{1}\left(j \omega_{i}\right)$ with different frequency variable at the end of the recursion. Thus they can be brought out as a common factor. This gives

$$
\varphi_{(p-1) n+1}\left(c_{p, 0}(\cdot)^{n} ; \omega_{l(1)} \cdots \omega_{l((p-1) n+1)}\right)=(-1)^{n} \prod_{i=1}^{(p-1) n+1} H_{1}\left(j \omega_{l(i)}\right) \cdot \varphi_{(p-1) n+1}^{\prime}\left(c_{p, 0}(\cdot)^{n} ; \omega_{l(1)} \cdots \omega_{l((p-1) n+1)}\right)
$$

where,

$$
\begin{aligned}
& \varphi_{(p-1) n+1}^{\prime}\left(c_{p, 0}(\cdot)^{n} ; \omega_{l(1)} \cdots \omega_{l((p-1) n+1)}\right) \\
& =\frac{-1}{L_{(p-1) n+1}\left(j \omega_{l(1)}+\cdots+j \omega_{l((p-1) n+1)}\right)} \cdot \sum_{\begin{array}{l}
\text { all the different combinations } \\
\text { of }\left\{\bar{x}_{1}, \overline{\mathrm{x}}_{2}, \ldots, \overline{\mathrm{x}}_{\mathrm{p}}\right\} \text { satisfying } \\
\overline{\mathrm{x}}_{1}+\cdots+\overline{\mathrm{x}}_{\mathrm{p}}=n-1,0 \leq \overline{\mathrm{x}}_{\mathrm{i}} \leq \mathrm{n}-1
\end{array}}^{p} \varphi_{(p-1) \bar{x}_{i}+1}^{\prime}\left(c_{p, 0}(\cdot)^{\bar{x}_{i}} ; \omega_{l(\overline{\mathrm{X}}(i)+1)} \cdots \omega_{l\left(\overline{\mathrm{X}}(i)+(p-1) \bar{x}_{i}+1\right)}\right) \\
& \cdot \frac{n_{x}^{*}\left(\overline{\mathrm{x}}_{1}, \cdots, \overline{\mathrm{x}}_{\mathrm{p}}\right)}{n_{k}^{*}\left(\mathrm{k}_{1}, \cdots, \mathrm{k}_{\mathrm{p}}\right)} \cdot \sum_{\substack{\text { all the different } \\
\text { permutations of } \\
\left\{\mathrm{k}_{1}, \cdots, \mathrm{k}_{\mathrm{p}}\right\}}} \prod_{i=1}^{p}\left(j \omega_{l(\bar{X}(i)+1)}+\cdots+j \omega_{l\left(\bar{X}(i)+(p-1) \bar{x}_{i}+1\right)}\right)^{k_{i}}
\end{aligned}
$$

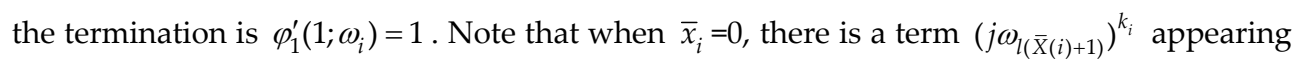

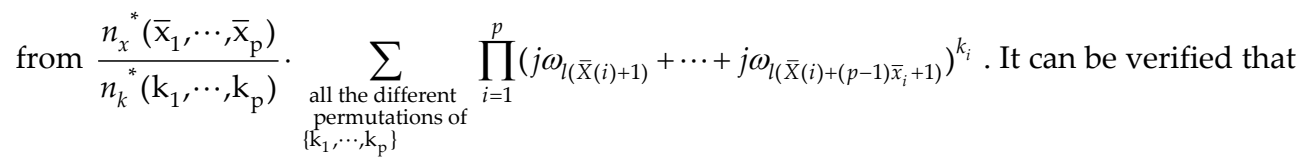
in each recursion of $\varphi_{(p-1) n+1}^{\prime}\left(c_{p, 0}(\cdot)^{n} ; \omega_{l(1)} \cdots \omega_{l((p-1) n+1)}\right)$, there may be some frequency variables appearing individually in the form of $\left(j \omega_{l\left(\bar{X}_{(i)+1)}\right.}\right)^{k_{i}}$, and these variables will not 
appear individually in the same form in the subsequent recursion. At the end of the recursion, all the frequency variables should have appeared in this form. Thus these terms can also be brought out as common factors if $k_{1}=k_{2}=\ldots=k_{\mathrm{p}}$. In the case of $k_{1}=k_{2}=\ldots=k_{\mathrm{p}}=k$,

$$
\begin{aligned}
& \frac{n_{x}^{*}\left(\overline{\mathrm{x}}_{1}, \cdots, \overline{\mathrm{x}}_{\mathrm{p}}\right)}{n_{k}^{*}\left(\mathrm{k}_{1}, \cdots, \mathrm{k}_{\mathrm{p}}\right)} \cdot \sum_{\substack{\text { all the different } \\
\text { permutations of } \\
\left\{\mathrm{k}_{1}, \cdots, \mathrm{k}_{\mathrm{p}}\right\}}} \prod_{i=1}^{p}\left(j \omega_{l(\bar{X}(i)+1)}+\cdots+j \omega_{l\left(\bar{X}(i)+(p-1) \bar{x}_{i}+1\right)}\right)^{k_{i}} \\
& =n_{x}{ }^{*}\left(\bar{x}_{1}, \cdots, \bar{x}_{\mathrm{p}}\right) \cdot \prod_{i=1}^{p}\left(j \omega_{l(\bar{X}(i)+1)}+\cdots+j \omega_{l\left(\bar{X}(i)+(p-1) \bar{x}_{i}+1\right)}\right)^{k_{i}}
\end{aligned}
$$

Therefore (A1) and (A2) can be written, if $k_{1}=k_{2}=\ldots=k_{\mathrm{p}}$, as

$$
\begin{aligned}
& \varphi_{(p-1) n+1}\left(c_{p, 0}(\cdot)^{n} ; \omega_{l(1)} \cdots \omega_{l((p-1) n+1)}\right) \\
& =(-1)^{n} \prod_{i=1}^{(p-1) n+1}\left[\left(j \omega_{l(i)}\right)^{k} H_{1}\left(j \omega_{l(i)}\right)\right] \cdot \varphi_{(p-1) n+1}^{\prime}\left(c_{p, 0}(\cdot)^{n} ; \omega_{l(1)} \cdots \omega_{l((p-1) n+1)}\right) \\
& \varphi_{(p-1) n+1}^{\prime}\left(c_{p, 0}(\cdot)^{n} ; \omega_{l(1)} \cdots \omega_{l((p-1) n+1)}\right) \\
& =\frac{-1}{L_{(p-1) n+1}\left(j \omega_{l(1)}+\cdots+j \omega_{l((p-1) n+1)}\right)} \cdot \sum_{\begin{array}{l}
\text { all the different combinations } \\
\text { of }\left\{\bar{X}_{1}, \bar{x}_{2}, \cdots, \bar{X}_{\mathrm{p}}\right\} \text { satisfying } \\
\overline{\mathrm{x}}_{1}+\cdots+\overline{\mathrm{p}}_{\mathrm{p}}=n-1,0 \leq \bar{x}_{\mathrm{i}} \leq n-1
\end{array}}^{p} \prod_{i=1}^{p} \varphi_{(p-1) \bar{x}_{i}+1}^{\prime}\left(c_{p, 0}(\cdot)^{\bar{x}_{i}} ; \omega_{l(\bar{X}(i)+1)} \cdots \omega_{l\left(\bar{X}(i)+(p-1) \bar{x}_{i}+1\right)}\right) \\
& \cdot n_{x}^{*}\left(\overline{\mathrm{x}}_{1}, \cdots, \overline{\mathrm{x}}_{\mathrm{p}}\right) \cdot \prod_{i=1}^{p}\left(j \omega_{l(\overline{\mathrm{X}}(i)+1)}+\cdots+j \omega_{l\left(\overline{\mathrm{X}}(i)+(p-1) \bar{x}_{i}+1\right)}\right)^{k_{i}\left(1-\delta\left(\bar{x}_{i}\right)\right)}
\end{aligned}
$$

(A4) can be further written as

$$
\begin{aligned}
& \varphi_{(p-1) n+1}^{\prime}\left(c_{p, 0}(\cdot)^{n} ; \omega_{l(1)} \cdots \omega_{l((p-1) n+1)}\right) \\
& =\frac{-1}{L_{(p-1) n+1}\left(j \omega_{l(1)}+\cdots+j \omega_{l((p-1) n+1)}\right)} \\
& \cdot \sum_{\substack{\text { all the different combinations } \\
\text { of }\left\{\bar{x}_{1}, \bar{x}_{2}, \ldots, \bar{x}_{\mathrm{p}}\right\} \text { satisfying } \\
\bar{x}_{1}, \bar{x}_{1}}} n_{x}{ }^{*}\left(\overline{\mathrm{x}}_{1}, \cdots, \overline{\mathrm{x}}_{\mathrm{p}}\right) \cdot \prod_{i=1}^{p} \varphi_{(p-1) \bar{x}_{i}+1}^{\prime \prime}\left(c_{p, 0}(\cdot)^{\bar{x}_{i}} ; \omega_{l(\bar{X}(i)+1)} \cdots \omega_{l\left(\bar{X}(i)+(p-1) \bar{x}_{i}+1\right)}\right)
\end{aligned}
$$

where, if $\bar{x}_{i}=0, \varphi_{(p-1) \bar{x}_{i}+1}^{\prime \prime}\left(c_{p, 0}(\cdot)^{\bar{x}_{i}} ; \omega_{l(\bar{X}(i)+1)} \cdots \omega_{l\left(\bar{X}(i)+(p-1) \bar{x}_{i}+1\right)}\right)=1$, otherwise, 


$$
\begin{aligned}
& \varphi_{(p-1) \bar{x}_{i}+1}^{\prime \prime}\left(c_{p, 0}(\cdot)^{\bar{x}_{i}} ; \omega_{l(\bar{X}(i)+1)} \cdots \omega_{l\left(\bar{X}(i)+(p-1) \bar{x}_{i}+1\right)}\right) \\
& =\left(j \omega_{l(\bar{X}(i)+1)}+\cdots+j \omega_{l\left(\bar{X}(i)+(p-1) x_{i}+1\right)}\right)^{k} \varphi_{(p-1) \bar{x}_{i}+1}^{\prime}\left(c_{p, 0}(\cdot)^{\bar{x}_{i}} ; \omega_{l(\bar{X}(i)+1)} \cdots \omega_{l\left(\bar{X}(i)+(p-1) \bar{x}_{i}+1\right)}\right) \\
& =\frac{\left(j \omega_{l(\bar{X}(i)+1)}+\cdots+j \omega_{l\left(\bar{X}(i)+(p-1) \bar{x}_{i}+1\right)}\right)^{k}}{-L_{(p-1) \bar{x}_{i}+1}\left(j \omega_{l(\bar{X}(i)+1)}+\cdots+j \omega_{l\left(\bar{X}(i)+(p-1) \bar{x}_{i}+1\right)}\right)} \cdot \sum_{\begin{array}{l}
\text { all the different combinations } \\
\text { of }\left\{x_{1}, x_{2}, \ldots, x_{p} p\right. \text { satitfying } \\
x_{1}+\cdots+x_{\mathrm{p}}=\bar{x}_{i}-1,0 \leq x_{\mathrm{i}} \leq \bar{x}_{i}-1
\end{array}} n_{x}^{*}\left(\mathrm{x}_{1}, \cdots, \mathrm{x}_{\mathrm{p}}\right) \\
& \cdot \prod_{i=1}^{p}\left(j \omega_{l\left(\bar{X}^{\prime}(i)+1\right)}+\cdots+j \omega_{l\left(\bar{X}^{\prime}(i)+(p-1) x_{i}+1\right)}\right)^{k_{i}\left(1-\delta\left(x_{i}\right)\right)} \varphi_{(p-1) x_{i}+1}^{\prime}\left(c_{p, 0}(\cdot)^{x_{i}} ; \omega_{l\left(\bar{X}^{\prime}(i)+1\right)} \cdots \omega_{l\left(\bar{X}^{\prime}(i)+(p-1) x_{i}+1\right)}\right) \\
& =\frac{\left(j \omega_{l(\bar{X}(i)+1)}+\cdots+j \omega_{l\left(\bar{X}(i)+(p-1) \bar{x}_{i}+1\right)}\right)^{k}}{-L_{(p-1) \bar{x}_{i}+1}\left(j \omega_{l(\bar{X}(i)+1)}+\cdots+j \omega_{l\left(\bar{X}(i)+(p-1) \bar{x}_{i}+1\right)}\right)} . \\
& \sum_{\substack{\text { all the different combinations } \\
\text { of }\left\{\mathrm{x}_{1}, \mathrm{x}_{2}, \ldots, \mathbf{X}_{\mathrm{p}}\right\} \text { satisfying }}} n_{x}^{*}\left(\mathrm{x}_{1}, \cdots, \mathrm{x}_{\mathrm{p}}\right) \cdot \prod_{i=1}^{p} \varphi_{(p-1) x_{i}+1}^{\prime \prime}\left(c_{p, 0}(\cdot)^{x_{i}} ; \omega_{l\left(\bar{X}^{\prime}(i)+1\right)} \cdots \omega_{l\left(\bar{X}^{\prime}(i)+(p-1) x_{i}+1\right)}\right) \\
& \text { of }\left\{\mathrm{x}_{1}, \mathrm{x}_{2}, \ldots, \mathrm{x}_{\mathrm{p}}\right\} \text { satisfying }
\end{aligned}
$$

The recursive terminal of (A6) is $\bar{x}_{i}=1$. Substituting (A2) into (A1) gives the first point of the lemma and substituting (A5) and (A6) into (A3) yields the first point of the lemma. This completes the proof.

Now proceed with the proof of Theorem 2. For convenience, denote

$$
\operatorname{sgn}_{c}\left(v_{1}\right)^{*} \operatorname{sgn}_{c}\left(v_{2}\right)=\operatorname{sgn}_{c}\left(v_{1} v_{2}\right)=\left[\operatorname{sgn}_{r}\left(\operatorname{Re}\left(v_{1} v_{2}\right)\right) \quad \operatorname{sgn}_{r}\left(\operatorname{Im}\left(v_{1} v_{2}\right)\right)\right]
$$

for any $v_{1}, v_{2} \in \mathbb{C}$.

Proof of Theorem 2. (1). From Lemma 1, any asymmetric $\varphi_{(p-1) n+1}\left(c_{p, 0}(\cdot)^{n} ; \omega_{k_{1}}, \cdots, \omega_{k_{(p-1) n+1}}\right)$ is sufficient for the computation of $\tilde{F}_{(p-1) n+1}(j \Omega)$. It can be obtained that

$$
\operatorname{sgn}_{c}\left(\tilde{F}_{(p-1) n+1}(j \Omega)\right)=\operatorname{sgn}_{c}\left(-j\left(\frac{F_{d}}{2}\right)^{(p-1) n+1}\right) * \operatorname{sgn}_{c}\left(\sum_{\omega_{k_{1}}+\cdots+\omega_{k_{(p-1) n+1}}=\Omega} \varphi_{(p-1) n+1}\left(c_{p, 0}(\cdot)^{n} ; \omega_{k_{1}}, \cdots, \omega_{k_{(p-1) n+1}}\right)\right)
$$

From Lemma 2, $\operatorname{sgn}_{c}\left(-j\left(\frac{F_{d}}{2}\right)^{(p-1) n+1}\right)$ has no effect on the alternating nature of the sequence $\tilde{F}_{(p-1) n+1}(j \Omega)$ for $n=1,2,3, \ldots$. Hence, $(21 \mathrm{a}-\mathrm{c})$ is an alternating series with respect to $c_{\mathrm{p}, 0}($.$) if and$ only if the sequence $\sum_{\omega_{k_{1}}+\cdots+\omega_{k_{(p-1) n+1}}=\Omega} \varphi_{(p-1) n+1}\left(c_{p, 0}(\cdot)^{n} ; \omega_{k_{1}}, \cdots, \omega_{k_{(p-1) n+1}}\right)$ for $n=1,2,3, \ldots$ is alternating. This is equivalent to

$$
\operatorname{sgn}_{c}\left(\sum_{\omega_{k_{1}}+\cdots+\omega_{k_{(p-1) n+1}}=\Omega}(-1)^{n-1} \varphi_{(p-1) n+1}\left(c_{p, 0}(\cdot)^{n} ; \omega_{l(1)} \cdots \omega_{l((p-1) n+1)}\right)\right)=\text { const }
$$


In the equation above, replacing $\varphi_{(p-1) n+1}\left(c_{p, 0}(\cdot)^{n} ; \omega_{k_{1}}, \cdots, \omega_{k_{(p-1) n+1}}\right)$ by using the result in Lemma 3 and noting $(p-1) n+1$ is an odd integer, it can be obtained that

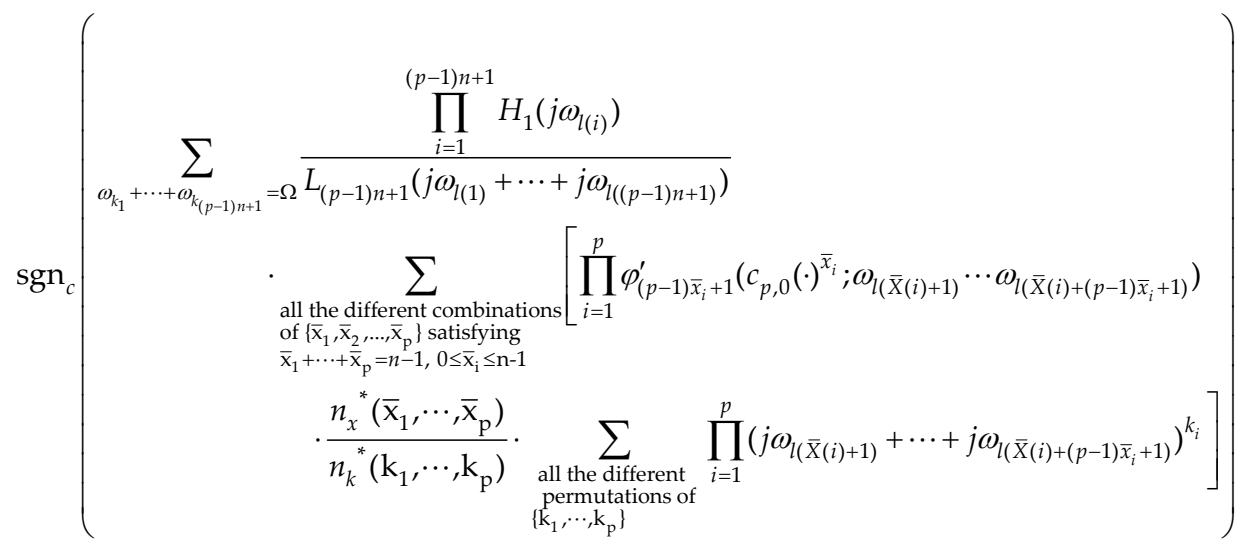

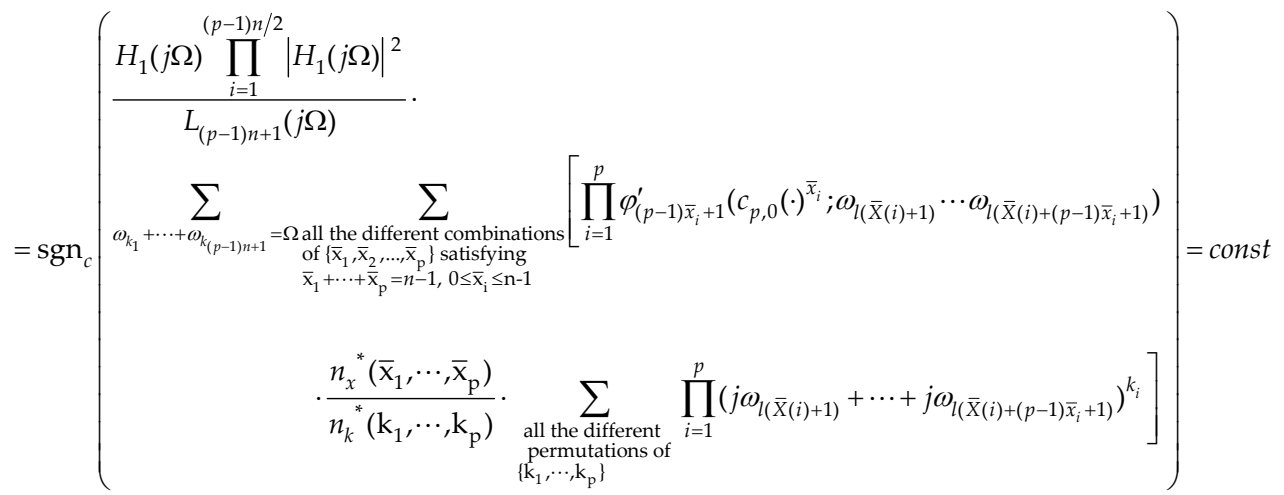

Note that $\prod_{i=1}^{(p-1) n / 2}\left|H_{1}(j \Omega)\right|^{2}$ has no effect on the equality above according to Lemma 2 , then the equation above is equivalent to (22).

(2). If additionally, $k_{1}=k_{2}=\ldots=k_{\mathrm{p}}=k$ in $c_{\mathrm{p}, 0}($.$) , then using the result in Lemma 3$, (22) can be written as 


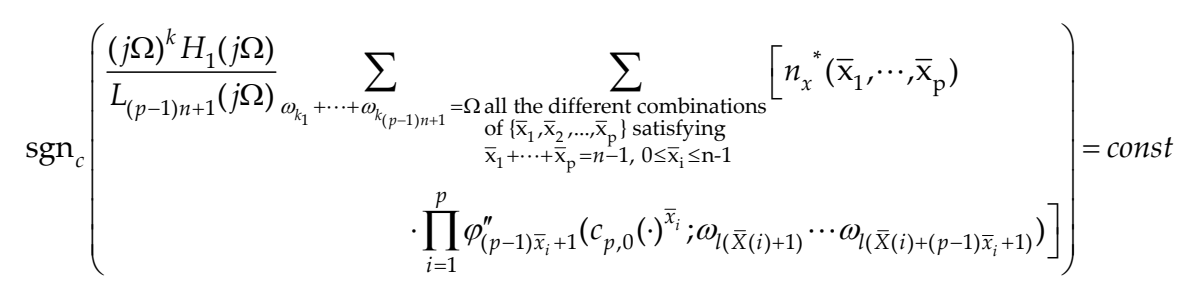

From Lemma 2, $(j \Omega)^{k}$ has no effect on this equation. Then the equation above is equivalent to

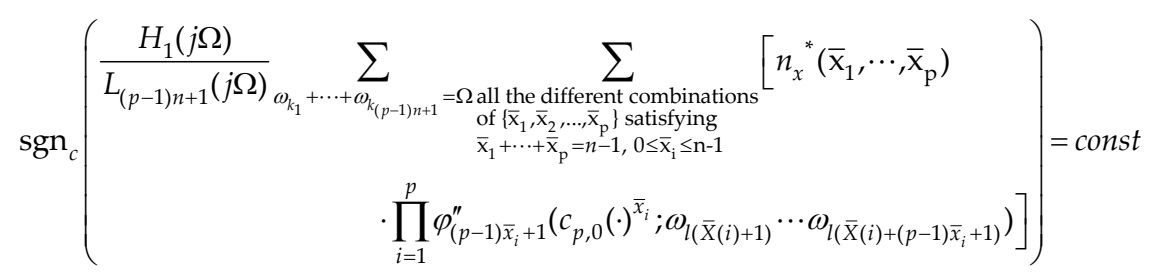

If $\operatorname{Re}\left(\frac{H_{1}(j \Omega)}{L_{(p-1) n+1}(j \Omega)}\right) \operatorname{Im}\left(\frac{H_{1}(j \Omega)}{L_{(p-1) n+1}(j \Omega)}\right)=0$, then $\frac{H_{1}(j \Omega)}{L_{(p-1) n+1}(j \Omega)}$ has no effect, either. This gives Equation (23). The proof is completed.

\section{References}

[1] Billings S.A. and Peyton-Jones J.C., “Mapping nonlinear integro-differential equation into the frequency domain", International Journal of Control, Vol 54, 863-879, 1990

[2] Boyd, S. and Chua L., "Fading memory and the problem of approximating nonlinear operators with Volterra series". IEEE Trans. On Circuits and Systems, Vol. CAS-32, No 11, pp 1150-1160, 1985

[3] Bromwich T. J., An Introduction to the Theory of Infinite Series, American Mathematical Society, AMS Chelsea Publishing, 1991

[4] George D.A., "Continuous nonlinear systems", Technical Report 355, MIT Research Laboratory of Electronics, Cambridge, Mass. Jul. 24, 1959.

[5] Graham D. and McRuer D., Analysis of nonlinear control systems. New York; London : Wiley, 1961.

[6] Jing X.J., Lang Z.Q., Billings S. A. and Tomlinson G. R., “The Parametric Characteristics of Frequency Response Functions for Nonlinear Systems", International Journal of Control, Vol. 79, No. 12, December, pp 1552 - 1564, 2006 
[7] Jing X.J., Lang Z.Q., and Billings S. A., "Mapping from parametric characteristics to generalised frequency response functions of nonlinear systems", International Journal of Control, 81(7), 1071-1088, 2008a

[8] Jing X.J., Lang Z.Q., Billings S. A. and Tomlinson G. R., Frequency domain analysis for suppression of output vibration from periodic disturbance using nonlinearities. Journal of Sound and Vibration, 314, 536-557, 2008b

[9] Lang Z.Q., and Billings S. A. "Output frequency characteristics of nonlinear systems". International Journal of Control, Vol. 64, 1049-1067, 1996

[10] Leonov G.A., Ponomarenko D.V. and Smirnova V.B. Frequency-domain methods for nonlinear analysis, theory and applications. World Scientific Publishing Co Pte Ltd, Singapore, 1996

[11] Ljung, L. System Identification: Theory for the User (second edition). Prentice Hall, Upper Saddle River. 1987 and 1999

[12] Pavlov A., van de Wouw N., and Nijmeijer H., Frequency Response Functions for Nonlinear Convergent Systems, IEEE Trans. Automatic Control, Vol 52, No 6, 11591165,2007

[13] Nuij P.W.J.M., Bosgra O.H., Steinbuch M. “Higher-order sinusoidal input describing functions for the analysis of non-linear systems with harmonic responses". Mechanical Systems and Signal Processing Vol 20, pp1883 - 1904, 2006

[14] Peyton-Jones J.C. "Simplified computation of the Volterra frequency response functions of nonlinear systems". Mechanical systems and signal processing, Vol 21, Issue 3, pp 1452-1468, April 2007

[15] Pintelon R. and Schoukens J., System Identification: A Frequency Domain Approach, IEEE Press, Piscataway, NJ, 2001

[16] Rugh W.J., Nonlinear System Theory: the Volterra/Wiener Approach, Baltimore, Maryland, U.S.A.: Johns Hopkins University Press, 1981

[17] Sandberg I. W., The mathematical foundations of associated expansions for mildly nonlinear systems, IEEE Trans. Circuits Syst., CAS-30, pp441-455, 1983

[18] Solomou, M. Evans, C. Rees, D. Chiras, N. “Frequency domain analysis of nonlinear systems driven by multiharmonic signals", Proceedings of the 19th IEEE conference on Instrumentation and Measurement Technology, Vol 1, pp: 799- 804, 2002

[19] Schetzen M., The Volterra and Wiener Theory of Nonlinear Systems, J. Wiley and Sons, 1980

[20] Volterra V., Theory of Functionals and of Integral and Integrodifferential Equations, Dover, New York, 1959

[21] Orlowski P., Frequency domain analysis of uncertain time-varying discretetime systems, Circuits Systems Signal Processing, Vol. 26, No. 3, 2007, PP. 293-310, 2007

[22] Jing X.J., Lang Z.Q., and Billings S. A., Parametric Characteristic Analysis for Generalized Frequency Response Functions of Nonlinear Systems. Circuits Syst Signal Process, DOI: 10.1007/s00034-009-9106-7, 2009 
34 Advances on Analysis and Control of Vibrations - Theory and Applications

[23] Jing X.J., Lang Z.Q., and Billings S. A., Determination of the analytical parametric relationship for output spectrum of Volterra systems based on its parametric characteristics, Journal of Mathematical Analysis and Application, 351, 694 - 706, 2009 